\title{
Model Predictive Control of an Underactuated Spacecraft with Two Reaction Wheels
}

\author{
Christopher D. Petersen* \\ University of Michigan, Ann Arbor, Michigan 48109-2140 \\ Frederick Leve‡ \\ U.S. Air Force Research Laboratory, Kirtland Air Force Base, Albuquerque, \\ New Mexico 87117-5776 \\ and \\ Ilya Kolmanovsky \\ University of Michigan, Ann Arbor, Michigan 48109-2140 \\ DOI: $10.2514 / 1 . G 000320$
}

\begin{abstract}
This paper presents an application of model predictive control to the attitude dynamics of an underactuated spacecraft with two reaction wheels and zero angular momentum. Such a system cannot be stabilized by any smooth or continuous time-invariant feedback law. However, model predictive control has a remarkable ability to generate control laws that are discontinuous in the state and, as such, can be applied to this problem. The model predictive control formulation is analyzed in depth and shown to produce an asymptotically stabilizing controller, which is discontinuous and enforces constraints. Simulations on the full nonlinear model demonstrate that model predictive control can successfully control the attitude of an underactuated spacecraft when the attitude maneuvers are sufficiently small.
\end{abstract}

$A$
$B$
$\mathcal{B}$
$F_{d}$
$f_{0}, f_{1}, f_{2}$
$\boldsymbol{H}$
$\mathcal{I}$
$\bar{J}$
$J_{N}$
$J_{s 1}, J_{s 2}$
$\bar{J}_{w 1}, \bar{J}_{w 2}$

$\bar{J}_{0}$

$L$
$\boldsymbol{M}_{\text {ext }}$
$\mathcal{O}_{\mathcal{B} / \mathcal{I}}$
$Q$
$r_{1}, r_{2}$
$T$
$\boldsymbol{u}$
$u_{\text {max }}$
$u_{1}, u_{2}$
$V_{N}$

\section{Nomenclature}

$=$ dynamics matrix of base variable linear model

$=$ input matrix of base variable linear model

$=$ spacecraft bus fixed frame

$=$ approximate attitude discrete dynamics

$=$ drift and control vector fields

$=$ inertial angular momentum vector expressed in $\mathcal{I}$

$=$ inertial frame corresponding to desired attitude

$=$ total inertia matrix

$=$ model predictive control objective function

$=$ inertias of reaction wheels 1 and 2 about their spin axes

$=$ inertia matrices of reaction wheels 1 and

2 relative to center of mass of spacecraft assembly and expressed in $\mathcal{B}$

$=$ inertia matrix of spacecraft bus relative to center of mass of spacecraft assembly and expressed in $\mathcal{B}$

$=$ model predictive control incremental cost

$=$ external moment vector expressed in $\mathcal{B}$

$=$ orientation matrix of $\mathcal{B}$ relative to $\mathcal{I}$

$=$ weighting matrix on state for incremental cost

$=$ weights on control for incremental cost

$=$ sample period for discrete dynamics

$=$ mathematical vector of control inputs corresponding to accelerations of reaction wheels

$=$ maximum limit on reaction wheel acceleration

$=$ components of $\boldsymbol{u}$

$=$ model predictive control value function

Received 30 November 2015; revision received 27 May 2016; accepted for publication 29 May 2016; published online 9 August 2016. Copyright @ 2016 by the American Institute of Aeronautics and Astronautics, Inc. All rights reserved. Copies of this paper may be made for personal and internal use, on condition that the copier pay the per-copy fee to the Copyright Clearance Center (CCC). All requests for copying and permission to reprint should be submitted to CCC at www.copyright.com; employ the ISSN 0731-5090 (print) or 1533-3884 (online) to initiate your request.

*Graduate Student, Department of Aerospace Engineering. Member AIAA.

${ }^{\dagger}$ Research Engineer, Guidance, Navigation, and Control Group.

FProfessor, Department of Aerospace Engineering. Member AIAA.

$\begin{array}{lll}W & = & \text { matrix of reaction wheel spin axes } \\ \hat{W} & = & \text { reaction wheel influence matrix } \\ \boldsymbol{w}_{1}, \boldsymbol{w}_{2} & = & \text { unit vectors of reaction wheel spin axes } \\ & & \text { expressed in } \mathcal{B} \\ \mathbb{X}_{k} & = & \text { state constraint set at discrete-time instant } k \\ \boldsymbol{x} & = & \text { vector of reduced variables } \phi, \theta, \psi, \nu_{1}, \nu_{2} \\ \boldsymbol{y} & = & \text { chosen base variable vector } \\ \hat{\boldsymbol{y}} & = & \text { components of } \hat{W} \\ \alpha_{1}, \alpha_{2}, \beta_{1}, \beta_{2} & \text { parameter for discrete change in } \psi \\ \Delta_{c} & = & \text { mathematical vector of Euler angles } \\ \boldsymbol{\Theta} & = & \text { mathematical vector of reaction wheels } \\ \nu & = & \text { velocities } \\ & & \text { respectities of reaction wheels } 1 \text { and } 2, \\ \nu_{1}, \nu_{2} & = & \text { angular velocity vector of } \mathcal{B} \\ \boldsymbol{\omega} & = & \text { relative to } \mathcal{I} \text { expressed in } \mathcal{B} \\ *_{k} & \end{array}$

\section{Introduction}

$\mathbf{M}$ ODEL predictive control (MPC) is a popular control technique due to its ability to generate feedback controllers that enforce specified constraints. Its application to the spacecraft attitude control problem began in the mid-1990s [1,2]. In [1], attitude tracking was performed using a one-step-ahead prediction of the states. On the other hand, [2] used a function-space MPC approach to track a reference attitude and differs from standard MPC because it does not recompute the optimization solution at every discrete-time step. Since then, the topic of MPC for spacecraft attitude has been approached in numerous other publications. In [3], a spacecraft with multiple thrusters and one reaction wheel (RW) is controlled by using explicit MPC. Gupta et al. [4] developed an MPC controller that acts on the manifold $\mathrm{SO}(3)$ to avoid mappings with singularities (such as Euler angles) and mappings that involve double covering (such as quaternions). Robust MPC for attitude control was discussed in $[\underline{5}, \underline{6}]$. MPC laws developed specifically for the case of control moment gyro and magnetic torque actuation are presented in [7-9] , respectively. An MPC algorithm suitable for fixed-point implementation is applied to spacecraft attitude control with RWs in [10].

The existing references on applications of MPC have not, however, addressed the case of a spacecraft that is underactuated by design or becomes underactuated as a result of onboard failures. It was shown in [11] that the attitude of a spacecraft can be controlled with less than 
three gas thrusters, whereas the spacecraft dynamics of a system with fewer than three RWs is inaccessible. Later, Krishnan et al. [12] proved that the attitude equations can be reduced for the case of zero angular momentum. These reduced equations are small-time locally controllable (STLC) from all orientations, which implies that arbitrary rest-to-rest reorientation maneuvers are possible. But due to Brockett's condition, an at-rest equilibrium cannot be stabilized by any time-invariant feedback law that is smooth or continuous in the state [12-15]. Time-periodic laws can be used for stabilization, but exponential convergence rates cannot be achieved if the feedback law is smooth [16]. Thus, although it is possible to control underactuated spacecraft attitude in an open-loop sense, there are inherent challenges in developing effective feedback stabilization solutions.

This paper approaches the attitude stabilization problem of a spacecraft with two RWs and zero angular momentum from an MPC standpoint. Several references have proposed non-MPC-type discontinuous control laws that successfully perform rest-to-rest attitude maneuvers for such an underactuated spacecraft (see [12, 17-22]). However, MPC has the remarkable ability to generate a stabilizing feedback law that is discontinuous as a function of the state $[23,24]$. In particular, this ability can be taken advantage of for nonholonomic system stabilization [25]. The use of MPC has several other advantages over other discontinuous feedback stabilization approaches: The design process is systematic, state and control constraints are handled, and a cost function reflecting performance objectives is optimized in a receding horizon sense.

The major contributions of this paper that address the underactuated spacecraft problem with two RWs and zero angular momentum are as follows: 1) the development of a set of reduced approximate equations of motion for the attitude dynamics in continuous-time and discrete-time; 2) a controllability analysis of the continuous-time, reduced approximate attitude dynamics and both a controllability and stabilizability analysis of the discrete-time, reduced approximate dynamics; 3 ) the introduction and analysis of a nonlinear MPC controller for the underactuated spacecraft problem, including showing that the control law generated using the reduced approximate dynamics is stabilizing. We also prove that the control law must be a nonsmooth function of the state and our numerical results indicate that the control law is actually a discontinuous function of the state; 4) the implementation of the nonlinear MPC controller on the full nonlinear underactuated spacecraft model, with discussion on real-time implementation of the controller.

Contributions 1 and 2 are significant because they provide the underlying framework of the nonlinear MPC controller presented. Contribution 3 is the main point of the paper. The fact that MPC generates a feedback law that is discontinuous in the state is interesting because stabilizing, continuous time-invariant feedback laws do not exist. Note that, in other problems, the MPC feedback law is typically continuous in the state. For example, in linear-quadratic-type MPC, the control law is always piecewise affine and continuous [26]. Contribution 4 is a demonstration that the nonlinear MPC controller presented has the potential to locally stabilize the attitude of the underactuated spacecraft, while adhering to constraints, provided its initial orientation is in the vicinity of the target orientation (which can be guaranteed by less-accurate thruster-based attitude control).

The paper is organized as follows. In Sec. II, the equations of motion for spacecraft attitude dynamics are introduced. Approximate continuous-time and discrete-time dynamic models are derived in Secs. II.B and II.C, respectively. The controllability properties and stabilization obstructions for the approximate dynamics are highlighted in Sec. III. In Sec. IV, the MPC formulation is presented for these approximate dynamics, and asymptotic stability properties are analyzed. Simulation results on the full nonlinear model (without approximations) are given in Sec. $\underline{\text { }}$. Concluding remarks are made in Sec. VI. The appendix that follows reviews results that we rely on for the use of Lie brackets to determine local controllability.

\section{Spacecraft Modeling}

In this paper, a spacecraft configuration consisting of a bus and two RWs is considered. The equations of motion are defined with the help of two reference frames: 1) an inertial frame $\mathcal{I}$ with attached orthonormal coordinate system and with the origin at the center of mass (c.m.) of the total spacecraft assembly (including the spacecraft bus and RWs); and 2) a spacecraft bus body-fixed frame $\mathcal{B}$ with the origin at the c.m. of the total spacecraft assembly.

We note that we do not assume $\mathcal{B}$ is a principal frame. Without loss of generality, we also assume that frame $\mathcal{I}$ is aligned to coincide with the desired inertial pointing attitude. The angular velocity of $\mathcal{B}$ relative to $\mathcal{I}$ and expressed in $\mathcal{B}$ is denoted by $\boldsymbol{\omega}$. The RWs spin with velocities $\nu_{1}$ and $\nu_{2}$ about nonparallel axes defined by $\boldsymbol{w}_{1}$ and $\boldsymbol{w}_{2}$, which are fixed and expressed in $\mathcal{B}$.

The relevant spacecraft inertias are described as follows. The matrices $\bar{J}_{0}, \bar{J}_{w 1}$, and $\bar{J}_{w 2}$ denote the inertia matrices of the spacecraft bus, RW 1, and RW 2, respectively, each relative to the c.m. of the spacecraft assembly and expressed in $\mathcal{B}$. Furthermore, we let $J_{s 1}$ and $J_{s 2}$ denote the inertias of RW 1 and RW 2 about their respective spin axes corresponding to unit vectors $\boldsymbol{w}_{1}$ and $\boldsymbol{w}_{2}$.

\section{A. Equations of Motion}

The orientation of $\mathcal{B}$ relative to $\mathcal{I}$ is characterized by three successive rotations, defined by 3-2-1 Euler angles yaw $\psi$, pitch $\theta$, and roll $\phi$. It is assumed that the maneuvers being performed involve relatively small attitude adjustments near the desired pointing orientation, and therefore the singularities in the Euler angle attitude representation are not of concern. Let $\boldsymbol{\Theta}=\left[\begin{array}{lll}\phi & \theta & \psi\end{array}\right]^{T}$. The spacecraft kinematic equations, following from the derivations in [27], are

$$
\dot{\boldsymbol{\Theta}}=M(\boldsymbol{\Theta}) \boldsymbol{\omega}
$$

in which

$$
M(\Theta)=\frac{1}{\cos (\theta)}\left[\begin{array}{ccc}
\cos (\theta) & \sin (\phi) \sin (\theta) & \cos (\phi) \sin (\theta) \\
0 & \cos (\phi) \cos (\theta) & -\sin (\phi) \cos (\theta) \\
0 & \sin (\phi) & \cos (\phi)
\end{array}\right]
$$

Let $\boldsymbol{H}$ be the (inertial) angular momentum vector of the spacecraft resolved in $\mathcal{I}$. Then, $\boldsymbol{H}$ is related to $\omega, \nu_{1}$, and $\nu_{2}$ by

$$
\mathcal{O}_{\mathcal{B} / \mathcal{I}} \boldsymbol{H}=\bar{J} \boldsymbol{\omega}+\bar{W} \boldsymbol{\nu}
$$

in which

$$
\begin{aligned}
& \bar{J}=\bar{J}_{0}+\bar{J}_{w 1}+\bar{J}_{w 2}, \\
& \bar{W}=W J_{s}, \\
& W=\left[\begin{array}{ll}
\boldsymbol{w}_{1} & \boldsymbol{w}_{2}
\end{array}\right], \\
& J_{s}=\operatorname{diag}\left(J_{s 1}, J_{s 2}\right), \\
& \nu=\left[\begin{array}{ll}
\nu_{1} & \nu_{2}
\end{array}\right]^{T}, \\
& \mathcal{O}_{\mathcal{B} / \mathcal{I}}=\left[\begin{array}{ccc}
\cos (\theta) \cos (\psi) & \cos (\theta) \sin (\psi) & -\sin (\theta) \\
\sin (\phi) \sin (\theta) \cos (\psi)-\cos (\phi) \sin (\psi) & \sin (\phi) \sin (\theta) \sin (\psi)+\cos (\phi) \cos (\psi) & \sin (\phi) \cos (\theta) \\
\cos (\phi) \sin (\theta) \cos (\psi)+\sin (\phi) \sin (\psi) & \cos (\phi) \sin (\theta) \sin (\psi)-\sin (\phi) \cos (\psi) & \cos (\phi) \cos (\theta)
\end{array}\right]
\end{aligned}
$$


The matrix $\bar{J}$ is the total inertia of the spacecraft assembly about its c. $\mathrm{m}$. The matrix $\mathcal{O}_{\mathcal{B} / \mathcal{I}}$ specifies the orientation of frame $\mathcal{B}$ relative to $\mathcal{I}$. It follows from the angular momentum balance relation, as well as [12], that the dynamic equations of motion are

$$
\bar{J} \dot{\omega}=-\boldsymbol{\omega} \times\left(\mathcal{O}_{\mathcal{B} / \mathcal{I}} \boldsymbol{H}\right)-\bar{W} \dot{\nu}+\boldsymbol{M}_{\mathrm{ext}}
$$

in which $\boldsymbol{M}_{\text {ext }}$ is the vector of external moments exerted on the spacecraft about the c.m. of the spacecraft and expressed in $\mathcal{B}$. In this work, the RW accelerations are treated as the control inputs

$$
\dot{\nu}=\boldsymbol{u}
$$

We now make the assumption that there are no external moments acting on the spacecraft (i.e., $\boldsymbol{M}_{\text {ext }}=0$ ), which implies that the inertial angular momentum $\boldsymbol{H}$ remains constant for all time. Furthermore, we assume that $\boldsymbol{H}=0$ such that controllability results similar to [12] can be obtained.

The set of Eqs. (1), (ㅁ), and (6) under the given assumptions are uncontrollable (as stated in [27]). However, progress can be made considering a reduced set of equations that are controllable, as in [12]. Specifically, exploiting the fact that $\boldsymbol{H}=0$, Eq. (3) reduces to

$$
\omega=-\bar{J}^{-1} \bar{W} \nu=\hat{W} \nu
$$

The matrix $\hat{W}$ has two columns, which represent the influence each RW has on the spacecraft bus assembly. It can be observed that the columns of $\hat{W}$ are linearly independent because the columns of $\bar{W}$ are linearly independent and $\bar{J}$ is full rank. We now assume that the columns of $\hat{W}$ are orthogonal to the third axis of the coordinate system attached to $\mathcal{B}$. The matrix $\hat{W}$ then has the form

$$
\hat{W}=\left[\begin{array}{cc}
\alpha_{1} & \alpha_{2} \\
\beta_{1} & \beta_{2} \\
0 & 0
\end{array}\right]
$$

in which $\alpha_{1}, \alpha_{2}, \beta_{1}, \beta_{2} \in \mathbb{R}$. Substituting Eq. (ㅁ) into Eq. (1) yields a new set of kinematic equations that, together with Eq. (6), form the reduced set of attitude equations

$$
\begin{aligned}
& \dot{\phi}=\left(\alpha_{1} \nu_{1}+\alpha_{2} \nu_{2}\right)+\left(\beta_{1} \nu_{1}+\beta_{2} \nu_{2}\right) \sin (\phi) \tan (\theta), \\
& \dot{\theta}=\left(\beta_{1} \nu_{1}+\beta_{2} \nu_{2}\right) \cos (\phi), \\
& \dot{\psi}=\left(\beta_{1} \nu_{1}+\beta_{2} \nu_{2}\right) \sin (\phi) \sec (\theta), \\
& \dot{\nu}_{1}=u_{1} \\
& \dot{\nu}_{2}=u_{2}
\end{aligned}
$$

in which $\boldsymbol{u}=\left[\begin{array}{ll}u_{1} & u_{2}\end{array}\right]^{T}$. We note that any equilibrium of Eq. (9) must be unforced (i.e., $u_{1}=u_{2}=0$ ). Furthermore, because $M(\boldsymbol{\Theta})$ is invertible, $\nu_{1}=\nu_{2}=0$ at an equilibrium. Thus, all attitudes with zero RW velocity are equilibria of Eq. (9).

\section{B. Approximation of the Equations of Motion}

To simplify controller design, the dynamics in Eq. (9) are approximated by a set of equations that do not contain any trigonometric functions. This is done by first expanding the trigonometric functions in Eq. (9) by a Taylor series, yielding

$$
\begin{aligned}
& \dot{\phi}=\left(\alpha_{1} \nu_{1}+\alpha_{2} \nu_{2}\right)+\left(\beta_{1} \nu_{1}+\beta_{2} \nu_{2}\right)\left(\phi-\frac{1}{6} \phi^{3}+\frac{1}{120} \phi^{5}+\cdots\right) \\
& \left(\theta+\frac{1}{3} \theta^{3}+\frac{2}{15} \theta^{5}+\cdots\right) \\
& \dot{\theta}=\left(\beta_{1} \nu_{1}+\beta_{2} \nu_{2}\right)\left(1-\frac{1}{2} \phi^{2}+\frac{1}{24} \phi^{4}+\cdots\right), \\
& \dot{\psi}=\left(\beta_{1} \nu_{1}+\beta_{2} \nu_{2}\right)\left(\phi-\frac{1}{6} \phi^{3}+\frac{1}{120} \phi^{5}+\cdots\right) \\
& \left(1+\frac{1}{2} \theta^{2}+\frac{5}{24} \theta^{4}+\cdots\right) \\
& \dot{\nu}_{1}=u_{1}, \\
& \dot{\nu}_{2}=u_{2}
\end{aligned}
$$

Equations (10) can be compactly written as

$$
\begin{aligned}
& \dot{\phi}=\left(\alpha_{1} \nu_{1}+\alpha_{2} \nu_{2}\right)+O\left(\|\boldsymbol{\Theta}(t)\|^{2}\right), \\
& \dot{\theta}=\left(\beta_{1} \nu_{1}+\beta_{2} \nu_{2}\right)+O\left(\|\boldsymbol{\Theta}(t)\|^{2}\right), \\
& \dot{\psi}=\left(\beta_{1} \nu_{1}+\beta_{2} \nu_{2}\right) \phi+O\left(\|\Theta(t)\|^{2}\right), \\
& \dot{\nu}_{1}=u_{1}, \\
& \dot{\nu}_{2}=u_{2}
\end{aligned}
$$

in which $O\left(\|\Theta(t)\|^{2}\right)$ denotes the remaining terms, which are higher order in Euler angles. Because the desired attitude maneuvers being performed are small, in a neighborhood of the desired pointing equilibrium $\Theta=0$, Eq. (9) can be approximated based on Eq. (11) by

$$
\begin{aligned}
& \dot{\phi}=\left(\alpha_{1} \nu_{1}+\alpha_{2} \nu_{2}\right), \\
& \dot{\theta}=\left(\beta_{1} \nu_{1}+\beta_{2} \nu_{2}\right), \\
& \dot{\psi}=\left(\beta_{1} \nu_{1}+\beta_{2} \nu_{2}\right) \phi, \\
& \dot{\nu}_{1}=u_{1}, \\
& \dot{\nu}_{2}=u_{2}
\end{aligned}
$$

Remark 1: When $\alpha_{1}=\beta_{2}=1$ and $\alpha_{2}=\beta_{1}=0$, the model (12) is essentially the same as the exact transformed equations of motion of the underactuated spacecraft with zero angular momentum given in [12]. This implies that the MPC law presented in this paper can also stabilize the transformed dynamics of [12], and hence the actual spacecraft's attitude. The advantage to using the transformed dynamics is that errors due to approximations like small angles are nonexistent. In this paper, the development of an MPC controller is based on model (12), and the transformation is not used to preserve the physical meaning and intuitive sense of state and control variables, which, for instance, facilitates the imposition of constraints. The controller is validated in simulations using the exact model based on Eqs. (1), (5), and (6).

Remark 2: The reduced, simplified continuous dynamics in model (12) are closely related to nonholonomic problems that have been studied in [28]. Although this paper only considers and focuses on the application of nonlinear MPC to the underactuated spacecraft problem, the results may be extended to stabilize other systems with similar characteristics. We leave such extensions to future work.

\section{Discretization}

To implement MPC, a discrete-time prediction model is needed. We assume that the control input is generated by a zero-order hold with a sampling period $T$ so that

$$
\begin{aligned}
& u_{1}(t)=u_{1, k}, \quad \forall t \in[k T,(k+1) T), \\
& u_{2}(t)=u_{2, k}, \quad \forall t \in[k T,(k+1) T)
\end{aligned}
$$


in which $k$ is a positive integer. In addition, we adopt the following notations:

$$
\begin{aligned}
& \phi_{k}=\phi(k T), \quad \theta_{k}=\theta(k T), \quad \psi_{k}=\psi(k T), \\
& \nu_{1, k}=\nu_{1}(k T), \quad \nu_{2, k}=\nu_{2}(k T), \\
& \boldsymbol{x}_{k}=\left[\begin{array}{lllll}
\phi_{k} & \theta_{k} & \psi_{k} & \nu_{1, k} & \nu_{2, k}
\end{array}\right]^{T}
\end{aligned}
$$

The discrete-time dynamics for RW velocities are determined by integrating the last two equations of Eqs. (11), yielding

$$
\nu_{1, k+1}=\nu_{1, k}+u_{1, k} T, \quad \nu_{2, k+1}=\nu_{2, k}+u_{2, k} T
$$

Based on model (12), the approximate discrete dynamics for Euler angles $\phi$ and $\theta$ can be determined similarly as

$$
\begin{aligned}
& \phi_{k+1}=\phi_{k}+\left(\alpha_{1} \nu_{1, k}+\alpha_{2} \nu_{2, k}\right) T+\left(\alpha_{1} u_{1, k}+\alpha_{2} u_{2, k}\right) \frac{T^{2}}{2} \\
& \theta_{k+1}=\theta_{k}+\left(\beta_{1} \nu_{1, k}+\beta_{2} \nu_{2, k}\right) T+\left(\beta_{1} u_{1, k}+\beta_{2} u_{2, k}\right) \frac{T^{2}}{2}
\end{aligned}
$$

To determine the approximate discrete dynamics for $\psi$, the equation

$$
\begin{aligned}
& \dot{\psi}=\left(\phi_{k}+\left(\alpha_{1} \nu_{1, k}+\alpha_{2} \nu_{2, k}\right) t+\left(\alpha_{1} u_{1, k}+\alpha_{2} u_{2, k}\right) \frac{t^{2}}{2}\right)\left(\beta_{1}\left(\nu_{1, k}+u_{1, k} t\right)\right) \\
& +\left(\phi_{k}+\left(\alpha_{1} \nu_{1, k}+\alpha_{2} \nu_{2, k}\right) t+\left(\alpha_{1} u_{1, k}+\alpha_{2} u_{2, k}\right) \frac{t^{2}}{2}\right)\left(\beta_{2}\left(\nu_{2, k}+u_{2, k} t\right)\right)
\end{aligned}
$$

must be integrated over the sampling period $T$, where Eq. (17) is obtained from substituting Eqs. (15) and (16) into the kinematic equation for $\psi$ in model (12). Integrating Eq. (17) and collecting Eqs. (15) and (16) gives the full set of approximate discrete dynamics

$$
\begin{aligned}
& \phi_{k+1}=\phi_{k}+\left(\alpha_{1} \nu_{1, k}+\alpha_{2} \nu_{2, k}\right) T+\left(\alpha_{1} u_{1, k}+\alpha_{2} u_{2, k}\right) \frac{T^{2}}{2}, \\
& \theta_{k+1}=\theta_{k}+\left(\beta_{1} \nu_{1, k}+\beta_{2} \nu_{2, k}\right) T+\left(\beta_{1} u_{1, k}+\beta_{2} u_{2, k}\right) \frac{T^{2}}{2}, \\
& \psi_{k+1}=\psi_{k}+\phi_{k}\left(\beta_{1} \nu_{1, k}+\beta_{2} \nu_{2, k}\right) T \\
& +\left(\beta_{1} \phi_{k} u_{1, k}+\beta_{2} \phi_{k} u_{2, k}+\alpha_{1} \beta_{1} \nu_{1, k}^{2}+\alpha_{2} \beta_{2} \nu_{2, k}^{2}\right. \\
& \left.+\alpha_{1} \beta_{2} \nu_{1, k} \nu_{2, k}+\alpha_{2} \beta_{1} \nu_{1, k} \nu_{2, k}\right) \frac{T^{2}}{2} \\
& +\left(3 \alpha_{1} \beta_{1} \nu_{1, k} u_{1, k}+3 \alpha_{2} \beta_{2} \nu_{2, k} u_{2, k}+2 \alpha_{1} \beta_{2} \nu_{1, k} u_{2, k}\right. \\
& \left.+2 \alpha_{2} \beta_{1} \nu_{2, k} u_{1, k}\right) \frac{T^{3}}{6}+\left(\alpha_{1} \beta_{2} \nu_{2, k} u_{1, k}+\alpha_{2} \beta_{1} \nu_{1, k} u_{2, k}\right) \frac{T^{3}}{6} \\
& +\left(\alpha_{1} \beta_{1} u_{1, k}^{2}+\alpha_{2} \beta_{2} u_{2, k}^{2}+\alpha_{1} \beta_{2} u_{1, k} u_{2, k}+\alpha_{2} \beta_{1} u_{1, k} u_{2, k}\right) \frac{T^{4}}{8}, \\
& \nu_{1, k+1}=\nu_{1, k}+u_{1, k} T \\
& \nu_{2, k+1}=\nu_{2, k}+u_{2, k} T
\end{aligned}
$$

Thus, under the assumption that all attitude maneuvers are relatively small, the discrete dynamics in model (18) approximate the actual discrete dynamics of the underactuated spacecraft system. We note that the approximate model (18) is nonlinear. The use of a nonlinear prediction model rather than linearized model is essential to be able to achieve discontinuous stabilization with MPC. This is because the linearized model is not controllable and thus a linear controller cannot be designed for this problem. In contrast, the simplified nonlinear model is locally controllable and stabilizable, which is discussed in the next section.

\section{Controllability and Stabilizability Analysis}

In this section, controllability and stabilizability properties of the approximate dynamics, given by the continuous-time equations in model (12) and the discrete-time equations in model (18), are analyzed and compared with the properties of the actual nonlinear dynamics (9). This analysis indicates that model (12) retains similar local controllability properties to Eq. (9), which are necessary to be able to use model (12) as a basis for control design for an underactuated spacecraft. Controllability and stabilizability properties of the discrete-time system (18) will subsequently be needed to demonstrate closed-loop stability with MPC.

\section{A. Controllability Analysis}

The definition of STLC, as given by [29], is as follows: A system $\dot{\boldsymbol{x}}=f(\boldsymbol{x}, \boldsymbol{u})$ is STLC from $\boldsymbol{x}_{0}$ if there exists a time $T>0$ such that, $\boldsymbol{x}_{0}$ for all time $t>0, t \leq T$ is in the interior of the reachable set from $x_{0}$, $R\left(x_{0}, t\right)$, at time instant $t$.

Intuitively, if $x_{0}$ is STLC, the reachable set from $x_{0}$ will remain an open neighborhood of $\boldsymbol{x}_{0}$ as time becomes infinitesimally small. In [12], the exact reduced underactuated attitude equations are proven STLC from any at-rest attitude. This property also holds for Eq. (9) (the proof of which is given in Appendix A). It is now demonstrated by Theorem 1 that the approximate continuous-time dynamics in model (12) remain STLC from any equilibrium. The proof of the theorem uses the concept of Lie brackets, which are reviewed in Appendix A.

Theorem 1: The approximate, underactuated spacecraft attitude Eqs. in (12) are STLC from all equilibria.

Proof: Let $\boldsymbol{x}=\left[\begin{array}{lllll}\phi & \theta & \psi & \nu_{1} & \nu_{2}\end{array}\right]^{T}$. The drift vector field and the control vector fields of model (12) are then given by

$$
\begin{aligned}
& f_{0}=\left[\begin{array}{lllll}
\alpha_{1} \nu_{1}+\alpha_{2} \nu_{2} & \beta_{1} \nu_{1}+\beta_{2} \nu_{2} & \left(\beta_{1} \nu_{1}+\beta_{2} \nu_{2}\right) \phi & 0 & 0
\end{array}\right]^{T}, \\
& f_{1}=\left[\begin{array}{lllll}
0 & 0 & 0 & 1 & 0
\end{array}\right]^{T} \text {, } \\
& f_{2}=\left[\begin{array}{lllll}
0 & 0 & 0 & 0 & 1
\end{array}\right]^{T}
\end{aligned}
$$

Using Eq. (A2) from Appendix A

$$
\begin{gathered}
B_{1}=\left[\begin{array}{ll}
f_{1}, & f_{0}
\end{array}\right]=\left[\begin{array}{lllll}
\alpha_{1} & \beta_{1} & \beta_{1} \phi & 0 & 0
\end{array}\right]^{T} \\
B_{2}=\left[\begin{array}{ll}
f_{2}, & f_{0}
\end{array}\right]=\left[\begin{array}{lllll}
\alpha_{2} & \beta_{2} & \beta_{2} \phi & 0 & 0
\end{array}\right]^{T} \\
B_{3}=\left[\begin{array}{lllll}
B_{1}, & B_{2}
\end{array}\right]=\left[\begin{array}{lllll}
0 & 0 & \left(\alpha_{1} \beta_{2}-\alpha_{2} \beta_{1}\right) & 0 & 0
\end{array}\right]^{T}
\end{gathered}
$$

Note the following:

1) The top two entries of $B_{1}$ and $B_{2}$ are equivalent to the top two entries of the first and second columns of $\hat{W}$, respectively. Because the columns of $\hat{W}$ are linearly independent, $B_{1}$ and $B_{2}$ are linearly independent for all $\phi$.

2) The top three entries of $B_{3}$ are equivalent to the cross product of the columns of $\hat{W}$. Because the columns of $\hat{W}$ are linearly independent, $B_{3}$ is nonzero.

From the preceding, it can be seen that the vector fields $f_{1}, f_{2}, B_{1}$, $B_{2}$, and $B_{3}$, when evaluated at any equilibrium (i.e., any orientation with zero RW velocity), span $\mathbb{R}^{5}$. By Theorem A1 in Appendix $\underline{A}$, model (12) is accessible from any equilibrium.

Now note that bracket $B_{3}$ has a 1 degree of four, the largest out of this set of brackets. The only bad brackets that can be generated by $f_{0}$, $f_{1}$, and $f_{2}$ with 1 degree less than four are $\left[f_{1}, B_{1}\right]$ and $\left[\begin{array}{ll}f_{2}, & B_{2}\end{array}\right]$, both of which have a 1 degree of three (other bad brackets of 1 degree of three can be written as linear combinations of these two due to the symmetric properties of the brackets themselves; see [30]). These brackets are zero and thus can be constructed trivially with good brackets of 1 degree of one or two. From Theorem A2 in Appendix A, the system is STLC from all equilibria.

The preceding analysis demonstrates that the approximate dynamics of model (12) retain the STLC property of the actual 
nonlinear dynamics (9). Thus, even though the system is approximate, its nonlinear dynamics can be exploited by control designs that depend on local controllability properties.

Remark 3: Consider now the discrete-time system (18). We note that there are accessibility properties that can be obtained for discretetime systems through the use of Lie brackets $[31,32]$, but to the authors' knowledge there is no Lie bracket analysis that can demonstrate nonlinear local controllability properties for discretetime that are similar to that of STLC in continuous-time. Hence, we present a direct construction of a control input that demonstrates the needed local controllability property in discrete-time in Appendix B. The open-loop control sequence that we construct brings any at-rest equilibrium of model (18) to any state in six steps, regardless of sample time. Thus, the reachable set from any attitude equilibrium in six steps is an open neighborhood. Moreover, the elements of the control sequence depend continuously on the initial state, a property that will be used in the proof of the closed-loop stability of the MPC controller.

\section{B. Stabilizability Analysis}

As stated previously, the actual continuous-time attitude dynamics cannot be stabilized by any smooth or continuous time-invariant feedback law due to Brockett's condition. This was proven in [12] and can be similarly shown for Eq. (9). To show that this obstruction to stabilization is still retained by model (18), the exact discretization of the reduced, approximate continuous-time dynamics in model (12), the following discrete-time variant of Brockett's condition from [33] is used.

Lemma 1[33]: Consider a discrete-time, nonlinear control system governed by

$$
\boldsymbol{x}_{k+1}=F_{d}\left(\boldsymbol{x}_{k}, \boldsymbol{u}_{k}\right)
$$

with $F_{d}(0,0)=0$ and $F_{d}$ being smooth (i.e., $\left.C^{\infty}\right)$ in a neighborhood of $(0,0)$. A necessary condition for the existence of a smooth state feedback control law $\boldsymbol{u}_{k}=\boldsymbol{u}\left(\boldsymbol{x}_{k}\right)$ that renders $(0,0)$ locally asymptotically stable is that the mapping $\Phi:(\boldsymbol{x}, \boldsymbol{u}) \rightarrow \boldsymbol{x}-F_{d}(\boldsymbol{x}, \boldsymbol{u})$ be onto an open neighborhood of the origin.

Lemma 1 is now used to prove the result of Theorem 2.

Theorem 2: There does not exist a smooth state feedback law that locally asymptotically stabilizes the discrete-time dynamics in model (18) to the origin.

Proof: If the map $\Phi$ is open, the equation

$$
z=x-F_{d}(x, u)
$$

is solvable for all $\boldsymbol{z}$ sufficiently small. Let $F_{d}$ represent the discrete dynamics in model (18) and $z=\left[\begin{array}{lllll}0 & 0 & \bar{z} & 0 & 0\end{array}\right]^{T}$ for $\bar{z} \in \mathbb{R}$. For Eq. (24) to be satisfied, $u_{1}, u_{2}, \nu_{1}$, and $\nu_{2}$ must be zero, which results in $\boldsymbol{x}-F_{d}(\boldsymbol{x}, \boldsymbol{u})=0$. Given that $W$ in Eq. (8) is rank 2, Eq. (24) is not solvable for all $|\bar{z}|>0$ and implies that the mapping $\boldsymbol{\Phi}$ is not open. The conclusion of the theorem follows from Lemma 1.

Theorem 2 demonstrates that, even though the dynamics are approximated and discretized, the obstruction to stabilizability present in Eq. (9) is retained by model (18). However, because the approximate nonlinear system is locally controllable, a nonlinear MPC controller can be used to generate a discontinuous feedback law to stabilize the system to the desired equilibrium.

\section{Model Predictive Control}

Model predictive control optimizes a control sequence over a finite horizon into the future to minimize a specified cost function subject to constraints [34]. Then, the first element of the optimal sequence is applied over the first discrete-time interval. The optimization horizon afterward recedes by one step and the process is repeated, starting with the current state as the initial condition.

Subsequently, we consider an MPC objective function for the spacecraft underactuated attitude problem of the form

$$
J_{N}\left(\boldsymbol{x}_{0}, u_{1,0}, \ldots, u_{1, N-1}, u_{2,0}, \ldots, u_{2, N-1}\right)=\sum_{i=0}^{N-1} L\left(\boldsymbol{x}_{i},\left[u_{1, i}, u_{2, i}\right]^{T}\right)
$$

in which $N$ is the optimization horizon and $L\left(\boldsymbol{x},\left[u_{1}, u_{2}\right]^{T}\right)$ is the incremental cost function given by

$$
L\left(\boldsymbol{x},\left[u_{1}, u_{2}\right]^{T}\right)=\boldsymbol{x}^{T} Q \boldsymbol{x}+r_{1} u_{1}^{2}+r_{2} u_{2}^{2}
$$

in which $Q=Q^{T}>0$ and $r_{1}, r_{2}>0$. The optimal control problem is given by

$$
\min _{u_{i, j}, i=1,2, j=0,1, \ldots, N-1} J_{N}\left(x_{0}, u_{1,0}, \ldots, u_{1, N-1}, u_{2,0}, \ldots, u_{2, N-1}\right)
$$

subject to

$$
\begin{aligned}
& \boldsymbol{x}_{k+1}=F_{d}\left(\boldsymbol{x}_{k},\left[u_{1, k}, u_{2, k}\right]^{T}\right), \quad \forall k=0,1, \ldots, N-1, \\
& \boldsymbol{x}_{0}=\boldsymbol{x}(t), \\
& \max \left\{\left|u_{1, k}\right|,\left|u_{2, k}\right|\right\} \leq u_{\max }, \quad k=0,1, \ldots, N-1, \\
& \boldsymbol{x}_{k} \in \mathbb{X}_{k}, \quad k=0,1, \ldots, N-1, \\
& \boldsymbol{x}_{N}=0
\end{aligned}
$$

in which $\boldsymbol{x}(t)$ is the current state, $u_{\max }$ is the maximum bound on control, $\mathbb{X}_{k}$ is the state constraint set at a discrete-time instant $k$ assumed to be compact, and $F_{d}$ are the approximate discrete dynamics in model (18). The value function of this optimization at $\boldsymbol{x}_{0}$ is defined as

$V_{N}\left(\boldsymbol{x}_{0}\right)=\min _{\boldsymbol{u}_{i, j}, i=1,2, j=0,1, \ldots, N-1} J_{N}\left(\boldsymbol{x}_{0}, u_{1,0}, \ldots, u_{1, N-1}, u_{2,0}, \ldots, u_{2, N-1}\right)$

\section{A. Asymptotically Stabilizing Control Generated by MPC}

To demonstrate that MPC generates an asymptotic stabilizing control law for the approximate discrete dynamics in model (18), the following theorem from [24] is used.

Theorem 3 [24]: For a discrete-time MPC problem with a terminal state condition $\boldsymbol{x}_{N}=0$, if $V_{N}$ is continuous at $\boldsymbol{x}_{0}=0$ and $L$ satisfies Requirements 3.1 and 3.2 , then the origin is an asymptotically stable equilibrium of the discrete-time system.

Requirement 3.1: $L(0,0)=0$

Requirement 3.2: There exists a nondecreasing function $\gamma:[0, \infty) \rightarrow[0, \infty)$ such that $\gamma(0)=0$ and $0<\gamma(\|(\boldsymbol{x}, \boldsymbol{u})\|) \leq$ $L(\boldsymbol{x}, \boldsymbol{u})$ for all $(\boldsymbol{x}, \boldsymbol{u}) \neq 0$, in which $\|(*, *)\|$ is a norm on the pair $(\boldsymbol{x}, \boldsymbol{u})$.

Note that Theorem 3 only requires continuity of the value function at $\boldsymbol{x}_{0}=0$. Theorem 3 is now used to prove that the nonlinear MPC problem generates an asymptotically stabilizing control law for when the input is unconstrained (Theorem 4) and constrained (Corollary 4.1).

Theorem 4: For the MPC problem in Eqs. (18), (27), and (28), let $\mathbb{X}_{k}=\mathbb{R}^{5}$ for $k=0,1, \ldots, N-1$ and $u_{\max }= \pm \infty$. Then, MPC generates an asymptotically stabilizing control law to the origin when the horizon length satisfies $N \geq 6$.

Proof: Requirements 3.1 and 3.2 are satisfied from the construction of $L$ in Eq. (26). It is now only necessary to show that $V_{N}$ is continuous at $x_{0}=0$ to apply Theorem 3. A sufficient condition for the continuity of $V_{N}$ at $x_{0}=0$ is that it is bounded from below and above by continuous functions that are zero at $\boldsymbol{x}_{0}=0$ [24]. The value function is bounded from below by a function because $\boldsymbol{x}^{\mathrm{T}} Q \boldsymbol{x}$. To show that $V_{N}$ is bounded from above, it is sufficient to show that an open-loop trajectory exists, is feasible under control 
constraints, and has a maneuver cost that is continuous in the initial state $\boldsymbol{x}_{0}$ and is zero at $\boldsymbol{x}_{0}=0$.

Such an open-loop control sequence can be constructed as follows. Let the states $\phi, \theta, \nu_{1}, \nu_{2}$ be called base variables, because the linear system consisting of these states is completely controllable in two discrete-time steps. Define $\boldsymbol{x}_{0}=\left[\begin{array}{lllll}\phi_{0} & \theta_{0} & \psi_{0} & \nu_{1,0} & \nu_{2,0}\end{array}\right]^{T}$ as the initial state vector, $\boldsymbol{y}_{0}=\left[\begin{array}{llll}\phi_{0} & \theta_{0} & \nu_{1,0} & \nu_{2,0}\end{array}\right]^{T}$ as the initial base variable vector, and $\hat{\boldsymbol{y}}=\left[\begin{array}{llll}\hat{\boldsymbol{\phi}} & \hat{\theta} & \hat{\nu}_{1} & \hat{\nu}_{2}\end{array}\right]^{T}$ as a chosen base variable vector. Then, the control sequence $\left\{u_{i, j}, i=1,2, j=0,1, \ldots, 5\right\}$, defined by

$$
\begin{gathered}
{\left[\begin{array}{llll}
u_{1,0} & u_{2,0} & u_{1,1} & u_{2,1}
\end{array}\right]^{T}=-\left(\left[\begin{array}{ll}
A B & B
\end{array}\right]\right)^{-1} A^{2} \boldsymbol{y}_{0}} \\
{\left[\begin{array}{llll}
u_{1,2} & u_{2,2} & u_{1,3} & u_{2,3}
\end{array}\right]^{T}=\left(\left[\begin{array}{ll}
A B & B
\end{array}\right]\right)^{-1} \hat{\boldsymbol{y}}} \\
{\left[\begin{array}{llll}
u_{1,4} & u_{2,4} & u_{1,5} & u_{2,5}
\end{array}\right]^{T}=-\left(\left[\begin{array}{ll}
A B & B
\end{array}\right]\right)^{-1} A^{2} \hat{\boldsymbol{y}}}
\end{gathered}
$$

and

$$
A=\left[\begin{array}{cccc}
1 & 0 & \alpha_{1} T & \alpha_{2} T \\
0 & 1 & \beta_{1} T & \beta_{2} T \\
0 & 0 & 1 & 0 \\
0 & 0 & 0 & 1
\end{array}\right], \quad B=\left[\begin{array}{cc}
\alpha_{1} \frac{T^{2}}{2} & \alpha_{2} \frac{T^{2}}{2} \\
\beta_{1} \frac{T^{2}}{2} & \beta_{2} \frac{T^{2}}{2} \\
T & 0 \\
0 & T
\end{array}\right]
$$

will drive any $\boldsymbol{x}_{0}$ to zero as long as

$$
\Delta_{c}=-\frac{12}{5 T} \psi_{2}
$$

in which

$$
\Delta_{c}=\hat{\phi}\left(\beta_{1} \hat{\nu}_{1}+\beta_{2} \hat{\nu}_{2}\right)-\hat{\theta}\left(\alpha_{1} \hat{\nu}_{1}+\alpha_{2} \hat{\nu}_{2}\right)
$$

and

$$
\begin{aligned}
\psi_{2} & =\psi_{0}+\frac{5 T}{24}\left(\phi_{0}\left(\beta_{1} \nu_{1,0}+\beta_{2} \nu_{2,0}\right)-\theta_{0}\left(\alpha_{1} \nu_{1,0}+\alpha_{2} \nu_{2,0}\right)\right) \\
& -\frac{1}{2} \theta_{0} \phi_{0}
\end{aligned}
$$

Note that, since $u_{\max }= \pm \infty$, the control in Eqs. (30-32) will always satisfy constraints, and therefore the trajectory generated by such a control sequence is feasible.

The logic of the open-loop maneuver as follows. The control sequence in Eq. (30) drives $\boldsymbol{y}_{0}$ to zero and $\psi_{0}$ to $\psi_{2}$. The remaining control sequences in Eqs. (31) and (32) guide the base variables in a closed trajectory that travels from zero to $\hat{\boldsymbol{y}}$ and back to zero. The influence of this closed trajectory on $\psi$ is reflected by $\psi_{3}, \psi_{4}, \psi_{5}$, and $\psi_{6}$ :

$$
\begin{gathered}
\psi_{3}=\psi_{2}+\Delta_{c} \frac{T}{16}+\hat{\theta} \hat{\phi} \frac{1}{8}+\left(\alpha_{1} \hat{\nu}_{1}+\alpha_{2} \hat{\nu}_{2}\right)\left(\beta_{1} \hat{\nu}_{1}+\beta_{2} \hat{\nu}_{2}\right) \frac{T^{2}}{32} \\
-\hat{\phi}\left(\beta_{1} \hat{\nu}_{1}+\beta_{2} \hat{\nu}_{2}\right) \frac{T}{8} \\
\psi_{4}=\psi_{2}+\Delta_{c} \frac{5 T}{24}+\frac{1}{2} \hat{\theta} \hat{\phi} \\
\psi_{5}=\psi_{2}+\Delta_{c} \frac{17 T}{48}+\hat{\theta} \hat{\phi} \frac{1}{8}+\left(\alpha_{1} \hat{\nu}_{1}+\alpha_{2} \hat{\nu}_{2}\right)\left(\beta_{1} \hat{\nu}_{1}+\beta_{2} \hat{\nu}_{2}\right) \frac{T^{2}}{32} \\
+\hat{\phi}\left(\beta_{1} \hat{\nu}_{1}+\beta_{2} \hat{\nu}_{2}\right) \frac{T}{8}
\end{gathered}
$$

$$
\psi_{6}=\psi_{2}+\Delta_{c} \frac{5 T}{12}
$$

As can be seen, if Eq. (34) is satisfied, $\psi_{6}=0$, and thus $\boldsymbol{x}_{0}$ reaches zero in six steps. Let $\hat{\boldsymbol{y}}$ be chosen as

$$
\begin{aligned}
& \hat{\phi}=\left|\psi_{2}\right|^{2 / 3}, \\
& \hat{\theta}=-\left|\psi_{2}\right|^{2 / 3}, \\
& \hat{\nu}_{1}=-\frac{6}{5 T\left(\alpha_{1}+\beta_{1}\right)}\left(\psi_{2}\right)^{1 / 3}, \\
& \hat{\nu}_{2}=-\frac{6}{5 T\left(\alpha_{2}+\beta_{2}\right)}\left(\psi_{2}\right)^{1 / 3}
\end{aligned}
$$

Then condition (34) is satisfied assuming that $\alpha_{1}+\beta_{1} \neq 0$ and $\alpha_{2}+\beta_{2} \neq 0$. Note if $\alpha_{1}+\beta_{1}=0$ or $\alpha_{2}+\beta_{2}=0$, another $\hat{\boldsymbol{y}}$ that satisfies condition (34) can be chosen. Also note that $\alpha_{1}+\beta_{1}=0$ and $\alpha_{2}+\beta_{2}=0$ will never occur at the same time because the columns of $\hat{W}$ are linearly independent. Using Eq. (41), the control sequences in Eqs. (31) and (32) become continuous functions of $\psi_{2}$, which is consequently a continuous function of $\boldsymbol{x}_{0}$.

Because the control sequence (30-32) steers the state to the origin and is continuous as a function of the initial state $\boldsymbol{x}_{0}$, it follows that $V_{N}$ is upper bounded by the cost of this feasible control sequence, which is a continuous function of $\boldsymbol{x}_{0}$ and is zero at $\boldsymbol{x}_{0}=0$. Because $V_{N}$ is upper and lower bounded by continuous functions of $\boldsymbol{x}_{0}$ that are zero at $\boldsymbol{x}_{0}=0, V_{N}$ is continuous at $\boldsymbol{x}_{0}=0$. By Theorem 3 , the control law is asymptotically stabilizing for all horizon lengths satisfying $N \geq 6$.

The argument of Theorem 4 can be extended to demonstrate the following result under control constraints.

Corollary 4.1: For the MPC problem in Eqs. (18), (27), and (28), let $\mathbb{X}_{k}=\mathbb{R}^{5}$ for $k=0,1, \ldots, N-1$. Then, for any $u_{\max }>0$, there exists a sufficiently large horizon length $N^{*} \geq 6$ such that MPC generates a locally asymptotic stabilizing control to the origin when the horizon length satisfies $N \geq N^{*}$.

Proof: Define the following quantities:

$$
\tilde{u}_{\max , 1}=\max \left\{\left|u_{1,0}\right|,\left|u_{1,1}\right|,\left|u_{2,0}\right|,\left|u_{2,1}\right|\right\}
$$

$$
\tilde{u}_{\max , 2}=\max \left\{\left|u_{i, j}\right|, i=1,2, j=2,3,4,5\right\}
$$

and the following set

$$
G(\rho)=\left\{\boldsymbol{x}_{0} \in \mathbb{R}^{5}: \quad \forall\left\|\boldsymbol{x}_{0}\right\| \leq \rho, \tilde{u}_{\max , 1} \leq u_{\max }\right\}
$$

Because the base variable system is linear [defined by matrices $A$ and $B$ from Eq. (33)] and $u_{\max }>0$, there exists a $\rho^{*}>0$ such that $\forall \rho \in\left(0, \rho^{*}\right], G \overline{(\rho)} \neq\{\varnothing\}$ (i.e., there exists a ball of radius $\rho^{*}$ centered at the origin where, for all $\boldsymbol{x}_{0}$ in the ball, $\left.\tilde{u}_{1, \max } \leq u_{\max }\right)$.

Now define $N_{\psi} \geq 1$ as a positive integer and let $\hat{\boldsymbol{y}}$ be chosen as

$$
\begin{aligned}
& \hat{\phi}=\left|\psi_{2}\right|^{2 / 3} / \sqrt{N_{\psi}}, \\
& \hat{\theta}=-\left|\psi_{2}\right|^{2 / 3} / \sqrt{N_{\psi}}, \\
& \hat{\nu}_{1}=-\frac{6}{5 T\left(\alpha_{1}+\beta_{1}\right) \sqrt{N_{\psi}}}\left(\psi_{2}\right)^{1 / 3}, \\
& \hat{\nu}_{2}=-\frac{6}{5 T\left(\alpha_{2}+\beta_{2}\right) \sqrt{N_{\psi}}}\left(\psi_{2}\right)^{1 / 3}
\end{aligned}
$$




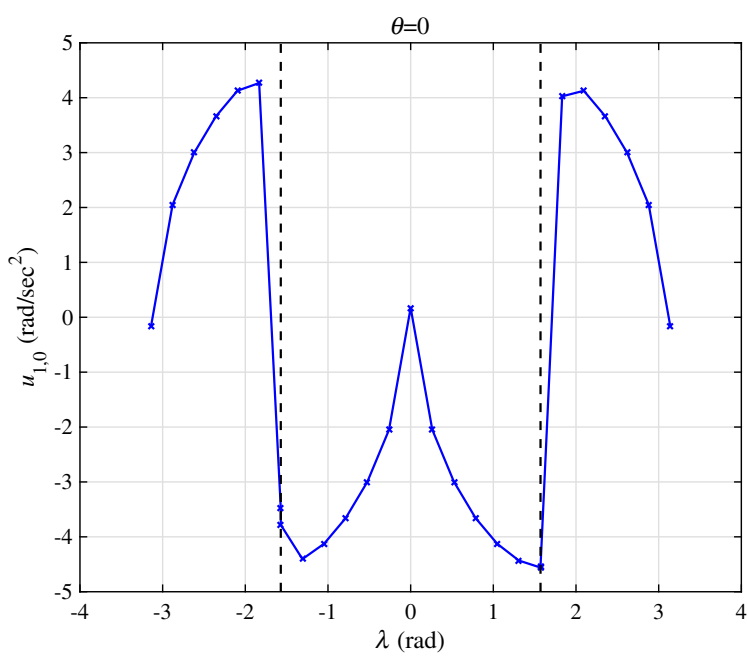

a) Control input $u_{1,0}$ vs $\lambda$ when $\theta=0$

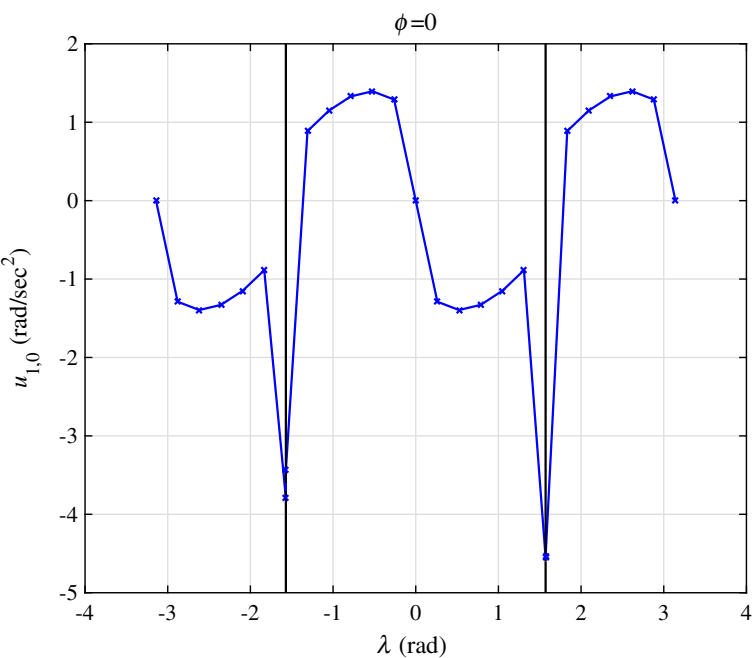

b) Control input $u_{1,0}$ vs $\lambda$ when $\phi=0$

Fig. 1 Discontinuous feedback law generated by MPC for a circle of initial conditions.

From the logic used in Theorem 4, if Eq. (ㄷ) holds, $\psi_{6}$ becomes

$$
\psi_{6}=\psi_{2}-\frac{\psi_{2}}{N_{\psi}}
$$

The control sequence $\left\{u_{i, j}, i=1,2, j=2,3,4,5\right\}$ can be repeated $n$ times, which gives the following discrete evolution of $\psi$ :

$$
\psi_{2+4 n}=\psi_{2}-\frac{\psi_{2}}{N_{\psi}} n
$$

If $n=N_{\psi}, \psi$ and the base variables reach zero in finite time. As $N_{\psi}$ increases, $\|\hat{\boldsymbol{y}}\|$ decreases, which consequently decreases $\tilde{u}_{\max , 2}$. Therefore, there exists an $N_{\psi}^{*} \geq 1$ such that, for all $N_{\psi} \geq N_{\psi}^{*}$, $\tilde{u}_{\max , 2} \leq u_{\max }$.

From the preceding, it can be seen that, for all $x_{0}$ in a neighborhood of the origin defined by $G\left(\rho^{*}\right)$, the MPC problem with control constraints is feasible for any horizon lengths satisfying $N \geq$ $2+4 N_{\psi}^{*}=N^{*}$. The control sequence for this maneuver is continuous in the initial condition $\boldsymbol{x}_{0}$, and therefore the cost of such a maneuver is continuous in $\boldsymbol{x}_{0}$ and zero at $\boldsymbol{x}_{0}=0$. The cost of this maneuver constitutes an upper bound on the value function, and hence the conclusions of this corollary follow similar to Theorem 4, but only apply in a neighborhood of the origin defined by $G\left(\rho^{*}\right)$.

Remark 4: It may be possible to increase $\rho^{*}$ if the control sequence that drives $\boldsymbol{y}_{0}$ to zero is allowed to take longer than two discrete-time steps. Therefore, by increasing $N$ further, it may be possible to expand the local region of attraction $G\left(\rho^{*}\right)$ for the MPC law defined in Eqs. (18), (27), and (28).

Theorem 4 only approaches the subject of control constraints. MPC, however, also has the ability to enforce state constraints. Though no formal proof in this work is given for stabilization in the presence of state constraints, simulations in Sec. V show that MPC can indeed generate stabilizing feedback laws when some state constraints are included.

Recall that the discrete dynamics used in the MPC formulation are only approximate. It is surmised that the MPC controller using the approximate dynamics is able to stabilize the actual orientation of the underactuated spacecraft in a region where the model mismatch is small. Simulation results on the full nonlinear model (1), (5), and (6) in Sec. V demonstrate successful convergence in a neighborhood of the origin where Euler angles, and thus model mismatch between the exact and approximate models, is sufficiently small.

\section{B. Discontinuous Control Law}

To illustrate that MPC generates a control law that is discontinuous in terms of state, the optimization problem in Eqs. (18), (27), and (28) is solved for various initial attitudes ranging between -0.1 and $0.1 \mathrm{rad}$ and initial RW velocities of $0 \mathrm{rad} / \mathrm{s}$. The spacecraft and the controller in these tests have the same parameters as those in Sec. $\mathrm{V}$.

Figure 1a shows the control action $u_{1,0}$ when $\theta=0$ and $\phi$ and $\psi$ are sampled on a circle of radius $0.05 \mathrm{rad}$ (for a given $\lambda, \phi=$ $0.05 \cos (\lambda)$ and $\psi=0.05 \sin (\lambda))$. Likewise, Fig. $1 \mathrm{~b}$ shows the

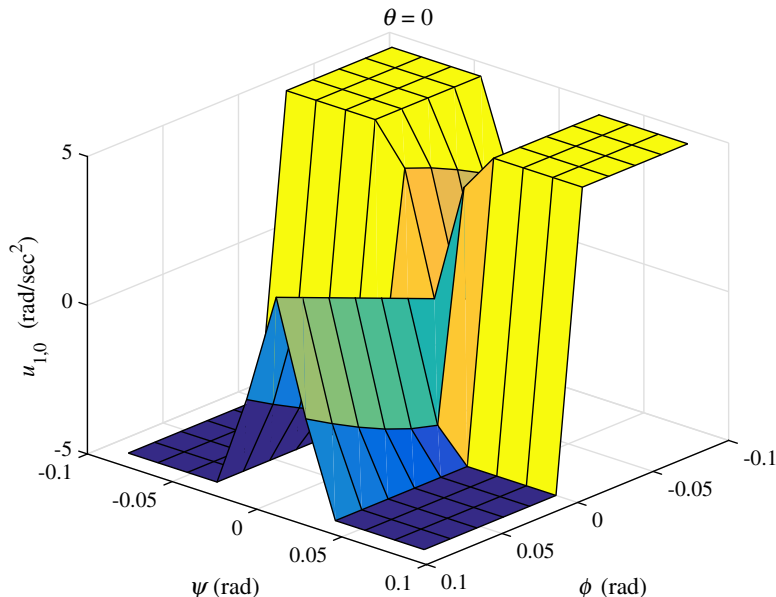

a)

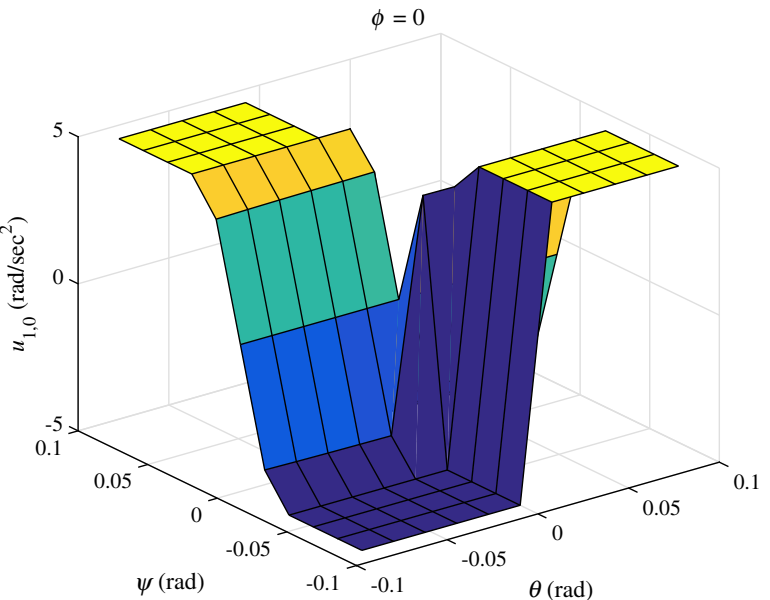

b)

Fig. 2 Discontinuous feedback law generated by MPC for a grid of initial conditions. 
Table 1 Simulation parameters

\begin{tabular}{lcc}
\hline \hline Parameter & Units & Value \\
\hline$u_{\max }$ & $\mathrm{rad} / \mathrm{s}^{2}$ & 5 \\
$T$ & $\mathrm{~s}$ & 10 \\
$N$ & -- & 30 \\
$Q$ & -- & $\operatorname{diag}\left(1 \times 10^{5}, 1 \times 10^{5}, 1 \times 10^{5}, 0.01,0.01\right)$ \\
$r_{1}, r_{2}$ & -- & 10,10 \\
\hline \hline
\end{tabular}

control action $u_{1,0}$ when $\phi=0$ and $\theta$ and $\psi$ are sampled on a circle of radius $0.05 \mathrm{rad}$, (for a given $\lambda, \theta=0.05 \cos (\lambda)$ and $\psi=0.05 \sin (\lambda))$. In both figures, the discontinuity, represented by the dashed line, occurs at $\phi=\theta=0$.

To demonstrate the discontinuity further, Fig. 2a shows the control action $u_{1,0}$ when $\theta=0$ and $\phi$ and $\psi$ are varied across a grid of initial conditions. Figure $2 \mathrm{~b}$ likewise shows the control action $u_{1,0}$ when $\phi=0$ and $\theta$ and $\psi$ are varied across a grid. The discontinuity is present and, in addition, passes through the origin. This can be reasoned from Fig. 1 as well, because as the radius of the sampled unit circle decreases, the discontinuity still remains at $\phi=\theta=0$, while $\psi$ decreases in magnitude.

\section{Simulation Results}

In this section, the MPC problem defined in Eqs. (18), (27), and (28) is applied to the actual nonlinear model (1), (5), and (6). The spacecraft bus in these simulations is assumed to have principal moments of inertia equal to 430,1210 , and $1300 \mathrm{~kg} \cdot \mathrm{m}^{2}$, respectively. The reaction wheels are assumed symmetric and thin, and they are mounted such that the c.m. of the spacecraft bus and total spacecraft assembly coincide. The inertias of the RWs about their spin axes are $J_{s 1}=J_{s 2}=0.043 \mathrm{~kg} \cdot \mathrm{m}^{2}$. The two RWs are aligned with the minor and intermediate principal axes of the spacecraft bus, yielding

$\bar{J}=\left[\begin{array}{ccc}430.043 & 0 & 0 \\ 0 & 1210.043 & 0 \\ 0 & 0 & 1300\end{array}\right], \quad \bar{W}=\left[\begin{array}{cc}0.043 & 0 \\ 0 & 0.043 \\ 0 & 0\end{array}\right]$

The model and control parameters used in all the simulations are listed in Table 1 .

\section{A. Beginning Simulations}

For the first simulation, the initial conditions of the spacecraft are $\boldsymbol{\Theta}(0)=\left[\begin{array}{lll}-0.05 & 0.03 & 0.1\end{array}\right]^{T} \mathrm{rad}, \boldsymbol{\omega}(0)=\left[\begin{array}{lll}0 & 0 & 0\end{array}\right]^{T} \mathrm{rad} / \mathrm{s}$, and $\nu(0)=\left[\begin{array}{ll}0 & 0\end{array}\right]^{T} \mathrm{rad} / \mathrm{s}$. In this simulation, only RW control constraints are enforced. The results are given in Fig. 3. In the second simulation, the initial conditions of the spacecraft are $\boldsymbol{\Theta}(0)=\left[\begin{array}{lll}0 & 0 & -0.1\end{array}\right]^{T} \mathrm{rad}, \boldsymbol{\omega}(0)=\left[\begin{array}{lll}0 & 0 & 0\end{array}\right]^{T} \mathrm{rad} / \mathrm{s}$, and $\boldsymbol{\nu}(0)=\left[\begin{array}{ll}0 & 0\end{array}\right]^{T} \mathrm{rad} / \mathrm{s}$. In this simulation, we impose an additional RW speed constraint of $\|\nu\|_{\infty} \leq 100 \mathrm{rad} / \mathrm{s}$. The results are given in Fig. 4. Both simulations demonstrate that the MPC formulation (27) and (28), which uses model (18) as an approximate model for prediction, is able to stabilize the attitude of the underactuated spacecraft to the desired pointing orientation, while enforcing control constraints on the exact model of the spacecraft. Moreover, the convergence rates in both simulations appear to be exponential.

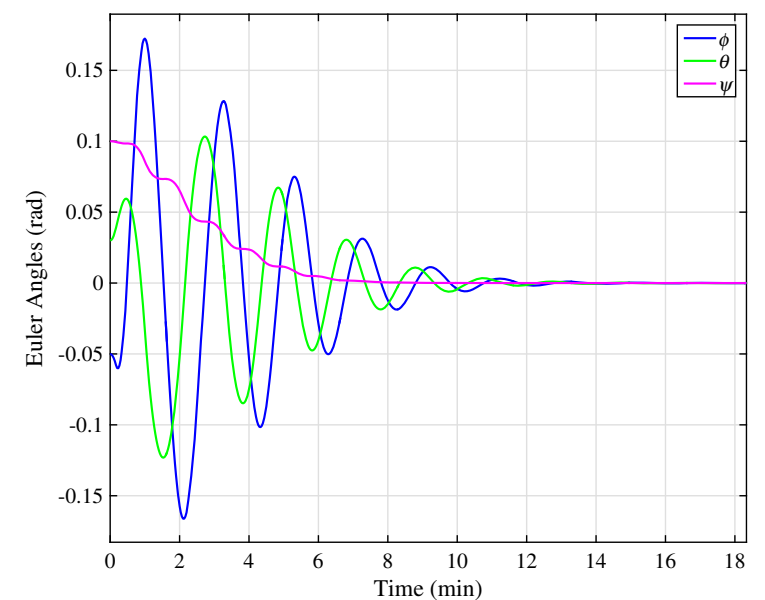

a)

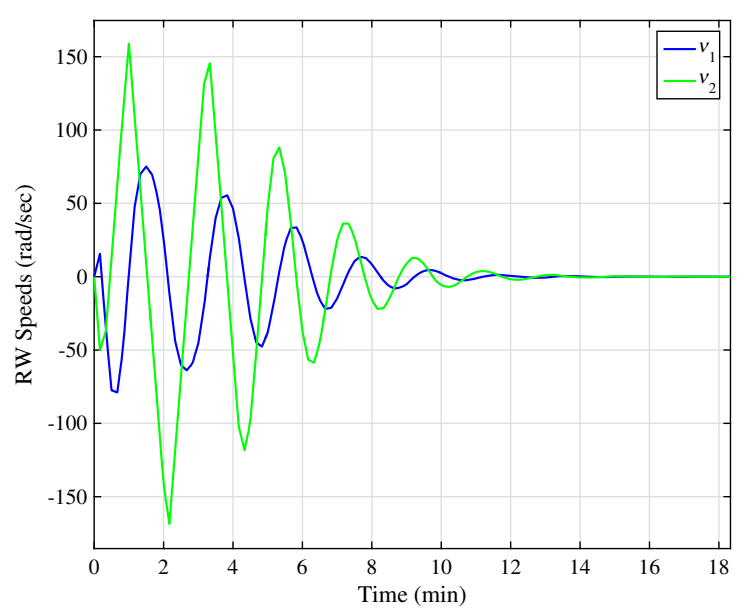

c)

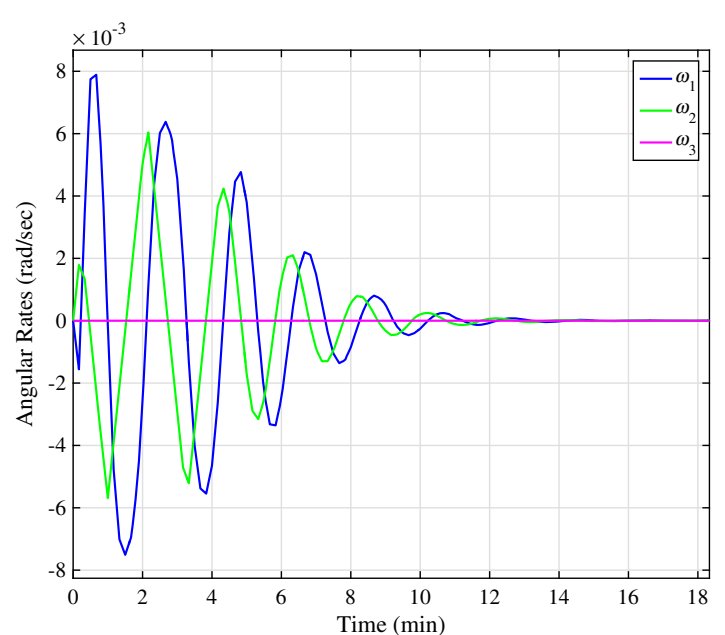

b)

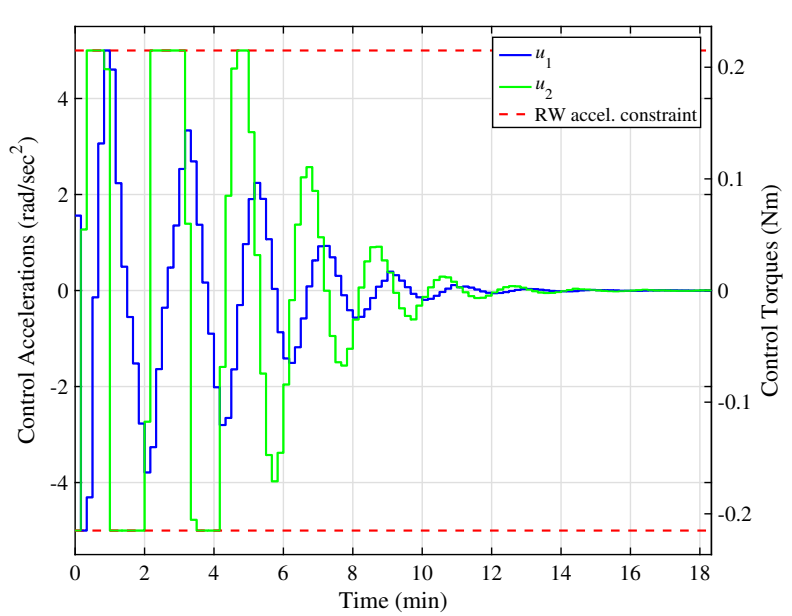

d)

Fig. 3 MPC response of underactuated spacecraft, simulation 1: a) Euler angles, b) angular velocities, c) wheel velocities, and d) wheel accelerations. 


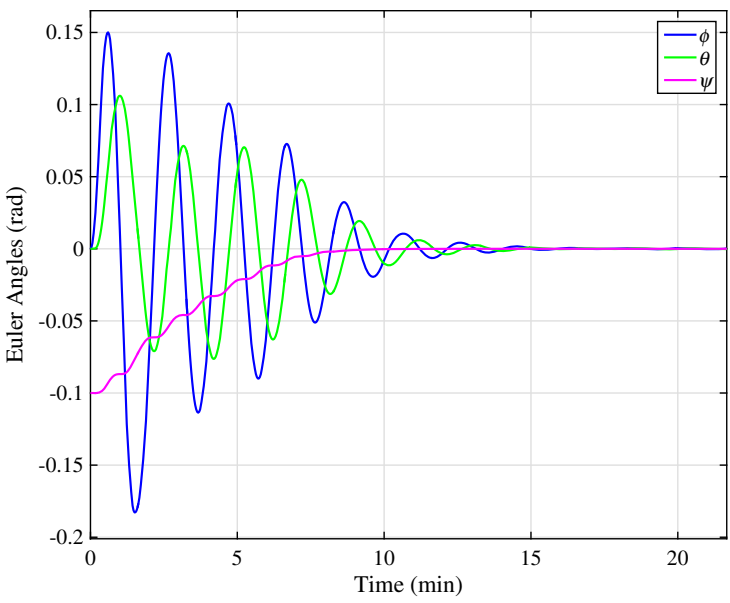

a)

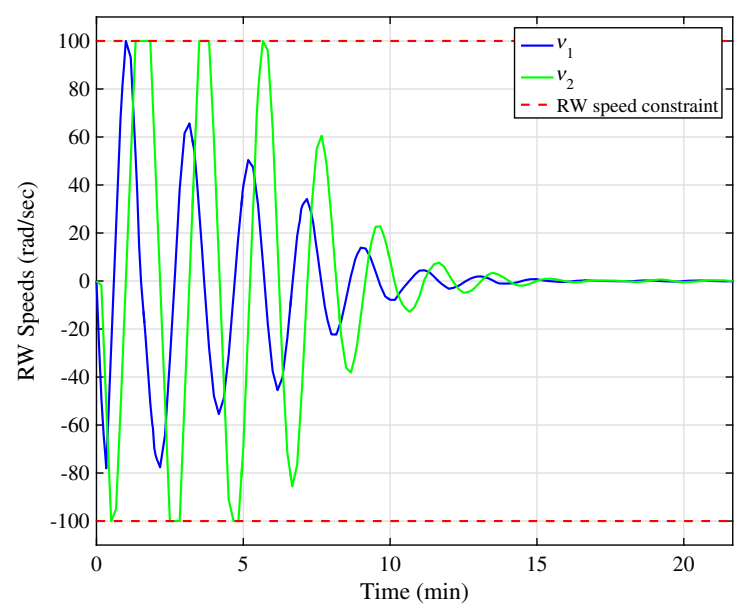

c)

Fig. 4 MPC response of underactuated spacecraft, simulation 2: a) Euler angl

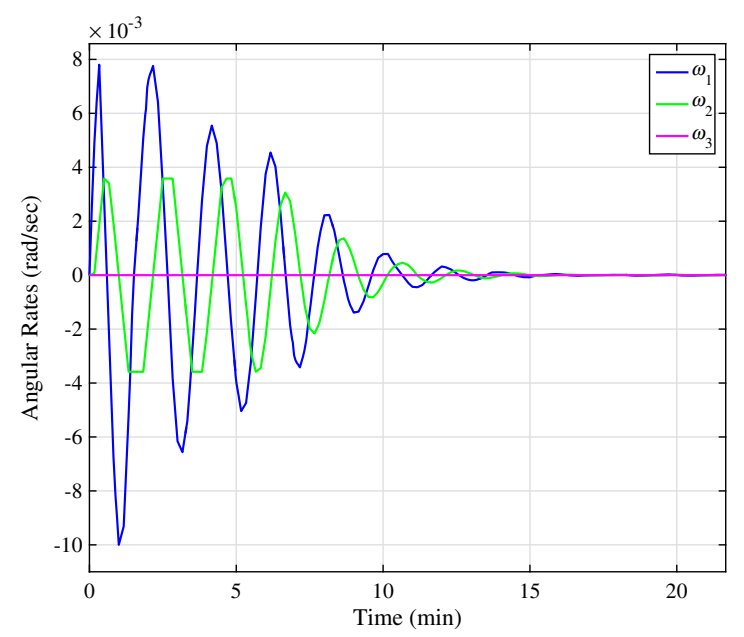

b)

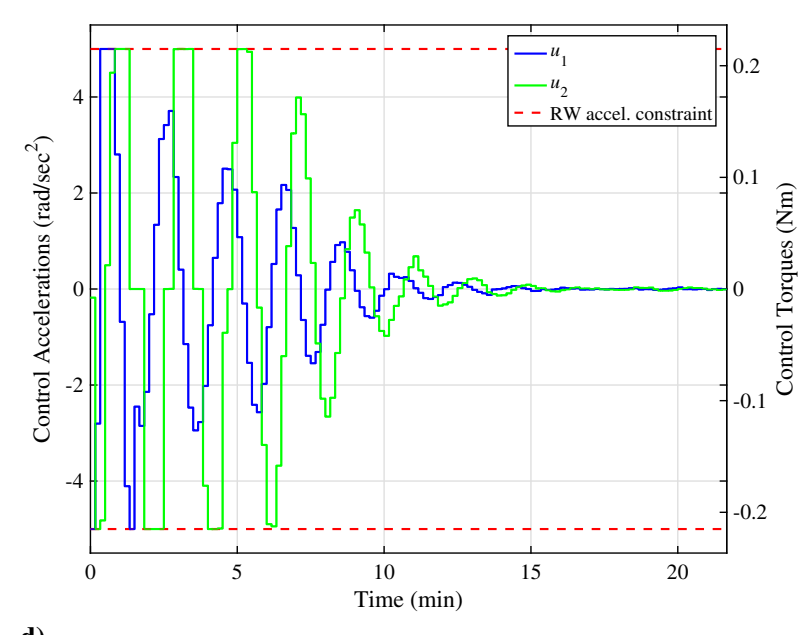

d)
Observe that, in both Figs. 3 and 4, the Euler angles $\phi$ and $\theta$ (roll and pitch) oscillate while $\psi$ (yaw) converges to equilibrium. For problems related to nonholonomic systems, such as the underactuated spacecraft problem, a controlled drift in the underactuated axis can be induced by performing oscillatory motion in the states that are controllable. This phenomenon is related to geometric phase, which is closely connected to the controllability analysis given by Lie brackets. Thus, it appears that MPC uses this effect to stabilize the underactuated spacecraft to equilibrium. An in-depth analysis of using geometric phase for the underactuated attitude problem can be found in $[\underline{35}, \underline{36}]$.

\section{B. Simulations with Various Sampling Times}

To demonstrate that the nonlinear MPC controller can handle different sampling periods, two simulations are now performed using the same initial conditions, control parameters, and constraints as the first simulation (results in Fig. 3 ), but the sampling period $T$ is changed from 10 to $6 \mathrm{~s}$ and $40 \mathrm{~s}$, respectively. The results are shown in Fig. 5. In both cases, the nonlinear MPC controller stabilizes the attitude, while satisfying constraints. Although this has not been done in simulations shown, the design parameters in Table 1, in particular, the prediction horizon, may need to be adjusted if the sampling period changes to improve closed-loop performance.

\section{Large Angle Maneuver Simulations}

As mentioned in the previous section, the nonlinear MPC controller is able to stabilize the attitude of the underactuated spacecraft in a neighborhood where model mismatch is small. This neighborhood can be easily reached by external thrusters or cold gas jets, types of actuation that can be used for large maneuvers but not for precise pointing. However, it can be demonstrated that the nonlinear MPC controller can also stabilize an at-rest spacecraft with initially large Euler angles. An example is given in Fig. 6, the control parameters used are in Table 1 , the initial conditions are $\boldsymbol{\Theta}(0)=\left[\begin{array}{lll}0.6 & -0.89 & 1\end{array}\right]^{T} \mathrm{rad}, \quad \boldsymbol{\omega}(0)=\left[\begin{array}{lll}0 & 0 & 0\end{array}\right]^{T} \mathrm{rad} / \mathrm{s}$, and $\nu(0)=\left[\begin{array}{ll}0 & 0\end{array}\right]^{T} \mathrm{rad} / \mathrm{s}$, and the only constraint being enforced is the control constraint. The figure demonstrates successful convergence while satisfying constraints, even though the smallangle assumption is clearly violated.

To further demonstrate the range of at-rest initial conditions the nonlinear MPC controller can stabilize, 1000 random test simulations were run with initial Euler angles belonging to the interval of $[-180,180] \mathrm{deg}$, initial zero angular velocity, and RW speeds initially at $0 \mathrm{rad} / \mathrm{s}$. Figure 7 gives an approximation of the region of attraction based on if the controller was able to converge to a $0.01 \mathrm{rad}$ $(0.573 \mathrm{deg})$ Euler angle box and a $0.001 \mathrm{rad} / \mathrm{s}$ angular velocity box. As can be seen, the region of attraction is quite large, despite the small-angle assumption being used in the controller design. Furthermore, the domain of attraction of the controller may be extended by exploiting reference governor strategies [37].

\section{Discussion on Real-Time Applications}

The MPC optimization problem in all simulations was solved using an interior-point method with MATLAB's fmincon function. The average and worst-case computation times needed to solve the optimization problem in Sec. V.A, using a standard computer with $2.4 \mathrm{GHz}$ clock speed, were 1.2 and $2.4 \mathrm{~s}$, respectively. Both times are less than the sample time $T$ in these simulations, which is $10 \mathrm{~s}$ (found 


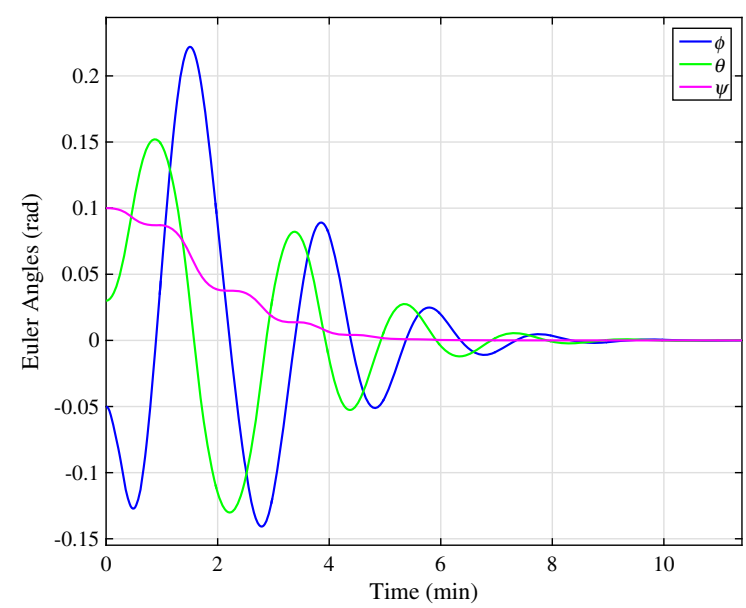

a)

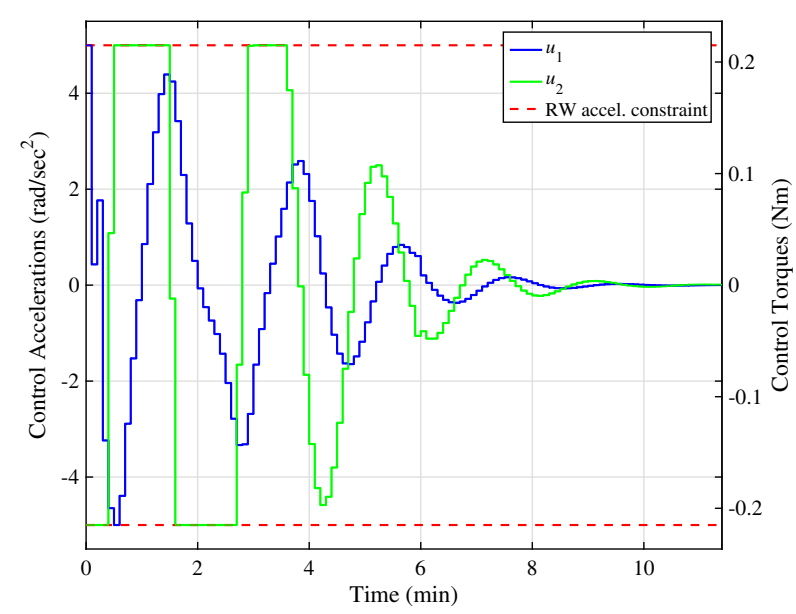

c)

Fig. 5 MPC response of underactuated spacecraft with various sample periods: Euler angles when a) $T=6 \mathrm{~s}$ and b) $T=40 \mathrm{~s}$; wheel accelerations when c) $T=6 \mathrm{~s}$ and d) $T=40 \mathrm{~s}$.

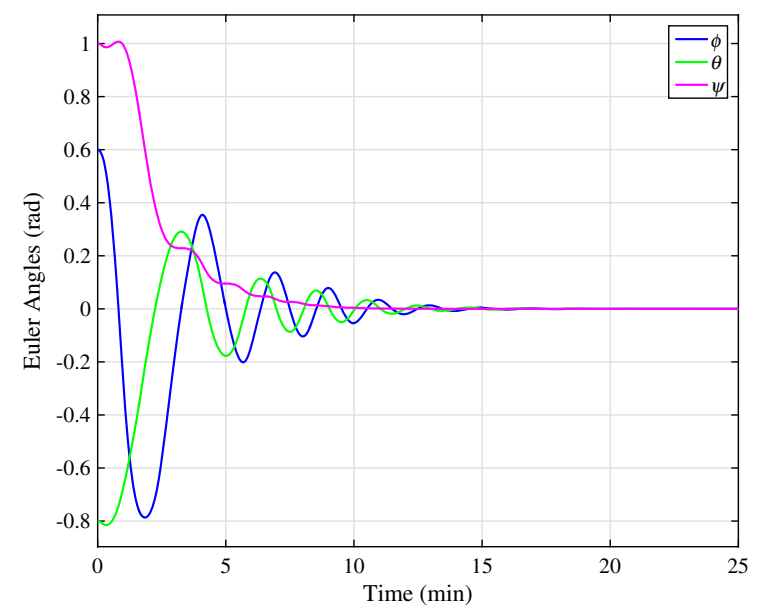

a)

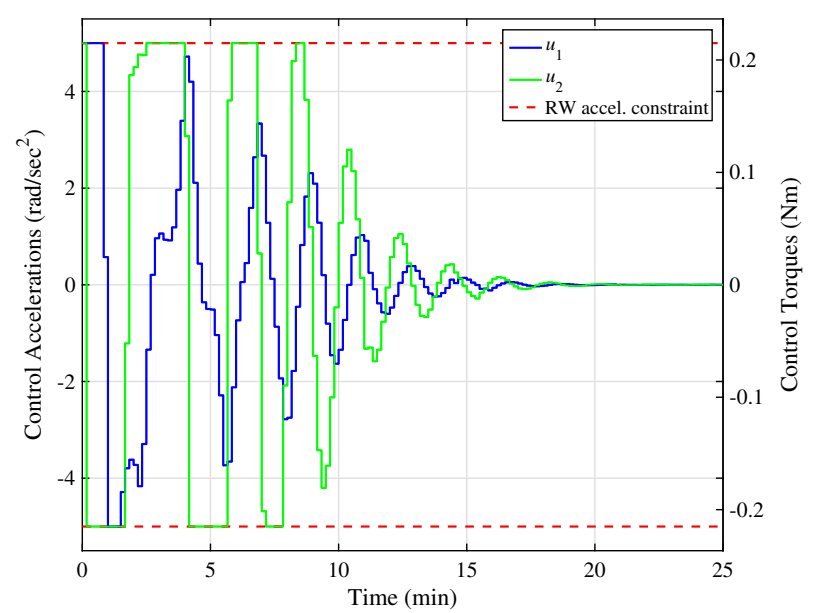

b)

Fig. 6 MPC response of underactuated spacecraft for large initial Euler angles: a) Euler angles and b) wheel accelerations.

in Table 1). Using custom solvers optimized for real-time implementation as a $C$ code will clearly reduce computation time. For instance, see [38], which shows that symbolic computations and code optimization can drastically improve the computation time. Though common spaceflight hardware have processing power typically in the $\mathrm{MHz}$ range, the general trend has been toward growing computing power. In fact, there are now more powerful

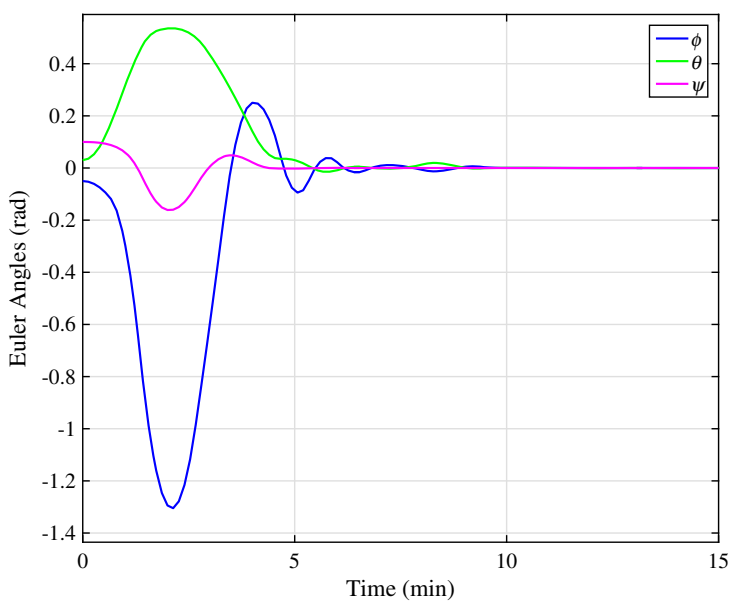

b)

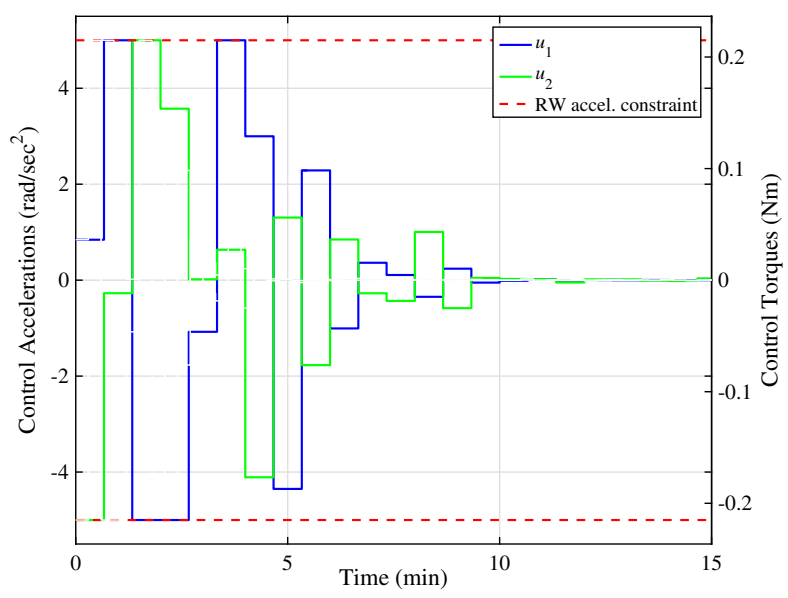

d) 


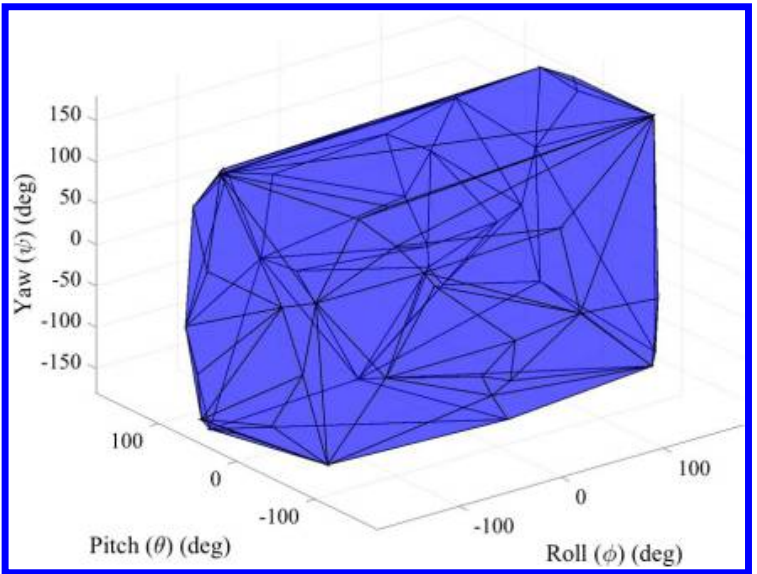

Fig. 7 Approximation of nonlinear MPC region of attraction for at-rest maneuvers.

for spacecraft missions with large processing demands [39]. It should finally be noted that RWs are used for nonagile maneuvers [40]. As such, the closed-loop bandwidth for actuation is in the range 0.01$1 \mathrm{~Hz}$ [41], hence control solutions do not need to be computed as rapidly as for other real-time systems. Thus, our application may not be dissimilar from other applications for which successful real-world implementations of nonlinear MPC have been reported $[42,43] . \mathbb{1}: * * *$ For spacecraft with limited onboard computational ability, an explicit implementation may be used in which the nonlinear MPC is precomputed offline and approximately function fitted; the fitted function is then used online [26,44-47]. Such an implementation is still fundamentally based on computational optimization.

Remark 5: Note that the underlying optimization problem is nonlinear and nonconvex. Thus, there are no a priori guarantees, other than offline testing by running multiple simulations, that the solver used will converge to a solution. Continuation and warm starting strategies can mitigate the risk of the solver not converging [47-49]. For some implementations, convergence is not required, only feasibility and cost decrease.

\section{Conclusions}

This paper analyzed the application of MPC to stabilize the attitude of an underactuated spacecraft with two RWs and zero angular momentum. It was shown that MPC based on an approximate model is able to generate a feedback law that is discontinuous in terms of state, which locally stabilizes the system to the desired pointing equilibrium. Simulations on the full nonlinear model demonstrate successful inertial pointing maneuvers with fast exponential convergence rates and with constraints being satisfied. The domain of attraction of the controller is an open neighborhood of the origin and it may be extended by exploiting reference governor strategies [37].

\section{Appendix A: Lie Brackets and Controllability}

Let a general control system for $\boldsymbol{x} \in \mathbb{R}^{n}$ that is affine in control be given by

$$
\dot{\boldsymbol{x}}=f_{0}(\boldsymbol{x})+\sum_{i=1}^{m} f_{i}(\boldsymbol{x}) u_{i}
$$

in which $u_{i}$ are the control inputs, $m$ is the number of control inputs, $f_{0}$ is the drift vector field, and $f_{i}, i=1, \ldots, m$ are the control vector fields. By studying the vector fields of Eq. (A1), as well as the vector fields generated by Lie brackets, certain controllability properties of Eq. (A1) can be obtained.

To begin, a Lie bracket of system (A1) is a bilinear skewsymmetric map that takes any two vector fields $f_{j}(\boldsymbol{x})$ and $f_{k}(\boldsymbol{x}), j$, $k \in\{0,1, \ldots, m\}$, and generates a third vector field on $\mathbb{R}^{n}$ in the following way $[\underline{12}, \underline{29}]$ :

$$
\left[f_{j}, f_{k}\right]=\frac{\partial f_{k}(\boldsymbol{x})}{\partial \boldsymbol{x}} f_{j}(\boldsymbol{x})-\frac{\partial f_{j}(\boldsymbol{x})}{\partial \boldsymbol{x}} f_{k}(\boldsymbol{x})
$$

Let $B$ denote any arbitrary Lie bracket of system (A1). Define the operator $|B|_{a}$ as the number of times the vector field $\overline{f_{a}}$ appears in the bracket, for any $a \in\{0,1, \ldots, m\}$. A bracket $B$ is bad if $|B|_{0}$ is odd and $|B|_{a}$ is even $\forall a \in 1,2, \ldots, m$. Otherwise, the bracket $B$ is good. Lastly, define the 1 degree operator of bracket $B$ as follows

$$
\operatorname{deg}_{1}(B)=\sum_{i=0}^{m}|B|_{i}
$$

The 1 degree operator is hence the total number of vector fields of Eq. (A1) used to generate Lie bracket $B$.

The following theorems summarize accessibility and controllability results found in $[29,30]$ and specializes them to the case of the system (A1). Refer to [29, $\underline{30]}$ for more general definitions.

Theorem A1: Let $\left(\boldsymbol{x}^{*}, \boldsymbol{u}^{*}\right)$ be an equilibrium of Eq. (A1). Define $D_{\infty}$ as the set of all vector fields in Eq. (A1) and all vector fields constructed from Lie brackets. The system Eq. (A1) is accessible from $\left(\boldsymbol{x}^{*}, \boldsymbol{u}^{*}\right)$ if and only if the $D_{\infty}$ spans $\mathbb{R}^{n}$ when the vector fields are evaluated at $\left(\boldsymbol{x}^{*}, \boldsymbol{u}^{*}\right)$. This is known as the Lie Algebra Rank Condition.

Theorem A2: Let $\left(\boldsymbol{x}^{*}, \boldsymbol{u}^{*}\right)$ be an equilibrium of Eq. (A1). Define $D_{\infty}$ as the set of all vector fields in Eq. (A1) and all vector fields constructed from Lie brackets. In addition, let $\hat{D} \subset D_{\infty}$ and $p$ be the largest 1 degree of all vector fields in $\hat{D}$ generated by Lie brackets. The system (A1) is STLC from $\left(\boldsymbol{x}^{*}, \boldsymbol{u}^{*}\right)$ if there exists a $\hat{D}$ such that the following conditions are met:

1) $\hat{D}$ satisfies the Lie Algebra Rank Condition when evaluated at $\left(\boldsymbol{x}^{*}, \boldsymbol{u}^{*}\right)$.

2) All bad brackets in $D_{\infty}$ of 1 degree less than $p$ when evaluated at $\left(\boldsymbol{x}^{*}, \boldsymbol{u}^{*}\right)$ are linear combinations of good brackets in $\hat{D}$ of lower order, when evaluated at $\left(\boldsymbol{x}^{*}, \boldsymbol{u}^{*}\right)$.

Note that Theorem A1 is sufficient and necessary, whereas Theorem A2 is only sufficient. To demonstrate these two theorems, the dynamics of Eq. (9) are analyzed using Lie brackets.

Theorem A3: The dynamics in Eq. (9) are STLC from any equilibria.

Proof: The drift vector field and the control vector fields of model (12) are

$$
\begin{aligned}
& f_{0}=\left[\begin{array}{c}
\left(\alpha_{1} \nu_{1}+\alpha_{2} \nu_{2}\right)+\left(\beta_{1} \nu_{1}+\beta_{2} \nu_{2}\right) \sin (\phi) \tan (\theta), \\
\left(\beta_{1} \nu_{1}+\beta_{2} \nu_{2}\right) \cos (\phi) \\
\\
\left(\beta_{1} \nu_{1}+\beta_{2} \nu_{2}\right) \sin (\phi) \sec (\theta) \\
0 \\
f_{1}
\end{array}=\left[\begin{array}{lllll}
0 & 0 & 0 & 1 & 0
\end{array}\right]^{T},\right. \\
& f_{2}=\left[\begin{array}{lllll}
0 & 0 & 0 & 0 & 1
\end{array}\right]^{T}
\end{aligned}
$$

Using Eq. (A2), three Lie brackets are generated:

$$
B_{1}=\left[f_{1}, f_{0}\right]=\left[\begin{array}{c}
\alpha_{1}+\beta_{1} \sin (\phi) \tan (\theta) \\
\beta_{1} \cos (\phi) \\
\beta_{1} \sin (\phi) \sec (\theta) \\
0 \\
0
\end{array}\right]
$$

\footnotetext{
"Data available online at http://www02.abb.com/global/gbabb/gbabb905. nsf/bf177942f19f4a98c1257148003b7a0a/4951ad3b82277aaec12579210051 feda/\$FILE/FFWD+Power+Generation+Special+Issue.pdf [retrieved 10 March 2016]

**Data available online at https://www.honeywellprocess.com [retrieved 10 March 2016].
} 


$$
\begin{gathered}
B_{2}=\left[f_{2}, f_{0}\right]=\left[\begin{array}{c}
\alpha_{2}+\beta_{2} \sin (\phi) \tan (\theta) \\
\beta_{2} \cos (\phi) \\
\beta_{2} \sin (\phi) \sec (\theta) \\
0 \\
0
\end{array}\right] \\
B_{3}=\left[B_{1}, B_{2}\right]=\left[\begin{array}{c}
\cos (\phi) \sin (\theta) \sec (\theta)\left(\alpha_{1} \beta_{2}-\alpha_{2} \beta_{1}\right) \\
-\sin (\phi)\left(\alpha_{1} \beta_{2}-\alpha_{2} \beta_{1}\right) \\
\cos (\phi) \sec (\theta)\left(\alpha_{1} \beta_{2}-\alpha_{2} \beta_{1}\right) \\
0 \\
0
\end{array}\right]
\end{gathered}
$$

Let $\hat{D}=\left\{f_{1}, f_{2}, B_{1}, B_{2}, B_{3}\right\}$. Note that $\hat{D}$ spans $\mathbb{R}^{5}$ when evaluated at any equilibrium, which implies $D_{\infty}$ spans $\mathbb{R}^{5}$ when evaluated at any equilibrium. By Theorem A1, the system is accessible from all equilibria.

It now is necessary to determine if the system is STLC from all equilibria. Note that, in $\hat{D}, B_{3}$ is constructed by using the drift vector $f_{0}$ twice and the control vectors $f_{1}$ and $f_{2}$ each once. Thus, the bracket is good and has a 1 degree of four, the largest of any other brackets in $\hat{D}$. The only bad brackets that can be constructed with a 1 degree less than four in $D_{\infty}$ are $\left[f_{1}, B_{1}\right]$ and $\left[f_{2}, B_{2}\right]$ (in the construction of each of these brackets, $f_{0}$ is used once and either $f_{1}$ or $f_{2}$ is used twice, and hence they are bad). These brackets are zero and thus are linear combinations of those brackets of 1 degree of two or less. By Theorem A2, the system is STLC from any equilibrium. $\square$

Note that the preceding proof is similar to the STLC analysis for the dynamics in model (12).

\section{Appendix B: Open-Loop Control Law for Approximate Attitude Dynamics}

Let $\boldsymbol{x}_{0}=\left[\begin{array}{lllll}\phi_{0} & \theta_{0} & \psi_{0} & 0 & 0\end{array}\right]^{T}$ be any equilibrium of model (18) and $\boldsymbol{x}^{*}=\left[\begin{array}{lllll}\phi^{*} & \theta^{*} & \psi^{*} & \nu_{1}^{*} & \nu_{2}^{*}\end{array}\right]^{T}$ be the desired state. Furthermore, define $\boldsymbol{y}_{0}=\left[\begin{array}{llll}\phi_{0} & \theta_{0} & 0 & 0\end{array}\right]^{T}$ as the initial base variable vector, $\boldsymbol{y}^{*}=\left[\begin{array}{llll}\phi^{*} & \theta^{*} & \nu_{1}^{*} & \nu_{2}^{*}\end{array}\right]^{T}$ as the desired base variable vector, and $\hat{\boldsymbol{y}}=\left[\begin{array}{llll}\hat{\phi} & \hat{\theta} & \hat{\nu}_{1} & \hat{\nu}_{2}\end{array}\right]^{T}$ as an arbitrary base variable vector. Then, the control sequence

$$
\begin{aligned}
& {\left[\begin{array}{llll}
u_{1,0} & u_{2,0} & u_{1,1} & u_{2,1}
\end{array}\right]^{T}=\left(\left[\begin{array}{ll}
A B & B
\end{array}\right]\right)^{-1}\left(\boldsymbol{y}^{*}-A^{2} \boldsymbol{y}_{0}\right)} \\
& {\left[\begin{array}{llll}
u_{1,2} & u_{2,2} & u_{1,3} & u_{2,3}
\end{array}\right]^{T}=\left(\left[\begin{array}{ll}
A B & B
\end{array}\right]\right)^{-1}\left(\hat{\boldsymbol{y}}-A^{2} \boldsymbol{y}^{*}\right)} \\
& {\left[\begin{array}{llll}
u_{1,4} & u_{2,4} & u_{1,5} & u_{2,5}
\end{array}\right]^{T}=\left(\left[\begin{array}{ll}
A B & B
\end{array}\right]\right)^{-1}\left(\boldsymbol{y}^{*}-A^{2} \hat{\boldsymbol{y}}\right)}
\end{aligned}
$$

in which

$$
A=\left[\begin{array}{cccc}
1 & 0 & \alpha_{1} T & \alpha_{2} T \\
0 & 1 & \beta_{1} T & \beta_{2} T \\
0 & 0 & 1 & 0 \\
0 & 0 & 0 & 1
\end{array}\right], \quad B=\left[\begin{array}{cc}
\alpha_{1} \frac{T^{2}}{2} & \alpha_{2} \frac{T^{2}}{2} \\
\beta_{1} \frac{T^{2}}{2} & \beta_{2} \frac{T^{2}}{2} \\
T & 0 \\
0 & T
\end{array}\right]
$$

will guide any $\boldsymbol{x}_{0}$ to $\boldsymbol{x}^{*}$ as long as

$$
\psi_{2}+\frac{5 T}{12} \Delta_{c}=\psi^{*}
$$

in which

$$
\begin{gathered}
\Delta_{c}=\left(\hat{\phi}-\phi^{*}\right)\left(\beta_{1}\left(\hat{\nu}_{1}-\nu_{1}^{*}\right)+\beta_{2}\left(\hat{\nu}_{2}-\nu_{2}^{*}\right)\right) \\
-\left(\hat{\theta}-\theta^{*}\right)\left(\alpha_{1}\left(\hat{\nu}_{1}-\nu_{1}^{*}\right)+\alpha_{2}\left(\hat{\nu}_{2}-\nu_{2}^{*}\right)\right)
\end{gathered}
$$

and

$$
\begin{aligned}
\psi_{2} & =\psi_{0}+\frac{5 T}{24}\left(\left(\phi^{*}-\phi_{0}\right)\left(\beta_{1} \nu_{1}^{*}+\beta_{2} \nu_{2}^{*}\right)\right. \\
& \left.-\left(\theta^{*}-\theta_{0}\right)\left(\alpha_{1} \nu_{1}^{*}+\alpha_{2} \nu_{2}^{*}\right)\right)+\frac{1}{2}\left(\theta^{*}-\theta_{0}\right)\left(\phi^{*}+\phi_{0}\right)
\end{aligned}
$$

Note that Eq. (B7) is the drift in the uncontrollable angle $\psi$ due to the control input $(\underline{\mathrm{B} 1})$. If Eq. ( $\underline{\mathrm{B} 5})$ is satisfied, $\boldsymbol{x}=\boldsymbol{x}^{*}$ in six steps. Now let

$$
\begin{aligned}
& \hat{\phi}=\phi^{*}+\sqrt{\left|\psi^{*}-\psi_{2}\right|}, \\
& \hat{\theta}=\theta^{*}-\sqrt{\left|\psi^{*}-\psi_{2}\right|}, \\
& \hat{\nu}_{1}=\frac{6}{5 T\left(\alpha_{1}+\beta_{1}\right)} \operatorname{sign}\left(\psi^{*}-\psi_{2}\right) \sqrt{\left|\psi^{*}-\psi_{2}\right|}+\nu_{1}^{*}, \\
& \hat{\nu}_{2}=\frac{6}{5 T\left(\alpha_{2}+\beta_{2}\right)} \operatorname{sign}\left(\psi^{*}-\psi_{2}\right) \sqrt{\left|\psi^{*}-\psi_{2}\right|}+\nu_{2}^{*}
\end{aligned}
$$

It can be seen that condition (34) is satisfied, assuming that $\alpha_{1}+\beta_{2} \neq 0$ and $\alpha_{2}+\beta_{2} \neq 0$ (this will not occur if the two RWs are not parallel, as mentioned in the proof of Theorem 4). If either $\alpha_{1}+\beta_{2} \neq 0$ or $\alpha_{2}+\beta_{2} \neq 0$, then other choices for $\hat{\boldsymbol{y}}$ do exist. Also note that this maneuver is possible for all sample times $T>0$ and only relies on the maneuvering being performed in six steps.

\section{References}

[1] Crassidis, J. L., Markley, F. L., Anthony, T. C., and Andrews, S. F., "Nonlinear Predictive Control of Spacecraft," Journal of Guidance, Control, and Dynamics, Vol. 20, No. 6, 1997, pp. 1096-1103. doi:10.2514/6.1997-114

[2] Wen, J. T., Seereeram, S., and Bayard, D. S., "Nonlinear Predictive Control Applied to Spacecraft Attitude Control," Proceedings of the American Control Conference, IEEE Publ., Piscataway, NJ, 1997, pp. 1899-1903. doi:10.1109/ACC.1997.610915

[3] Hegrenas, O., Gravdahl, J. T., and Tondel, P., "Attitude Control by Means of Explicit Model Predictive Control, via Multi-Parametric Quadratic Programming," Proceedings of the American Control Conference, IEEE Publ., Piscataway, NJ, 2005, pp. 901-906. doi:10.1109/ACC.2005.1470074

- [4] Gupta, R., Kalabic, U. V., Di Cairano, S., Bloch, A. M., and Kolmanovsky, I. V., "Constrained Spacecraft Attitude Control on SO(3) Using Fast Nonlinear Model Predictive Control," Proceedings of the American Control Conference, IEEE Publ, Piscataway, NJ, 2015, pp. 2980-2986. doi:10.1109/ACC.2015.7171188

[5] Kim, J., and Crassidis, J. L., "Spacecraft Attitude Control Using Approximate Receding-Horizon Model-Error Control Synthesis," Journal of Guidance, Control, and Dynamics, Vol. 29, No. 5, 2006, pp. 1023-1031. doi:10.2514/6.2005-6178

[6] Myung, H., and Bang, H., "Nonlinear Predictive Attitude Control of Spacecraft Under External Disturbances," Journal of Spacecraft and Rockets, Vol. 40, No. 5, 2003, pp. 696-699. doi: $10.2514 / 2.6896$

[7] Ikeda, Y., Nakajima, T., and Chida, Y., "Attitude Control of Spacecraft by NMPC with Consideration of Singularity Avoidance of CMG," Proceedings of the Conference on Decision and Control, IEEE Publ., Piscataway, NJ, 2012, pp. 1733-1739. doi:10.1109/CDC.2012.6426827

[8] Yi, $\overline{\text { C., and Wen-Hua, C., "Automatic Differentiation Based Nonlinear }}$ Model Predictive Control of Satellites Using Magneto-Torquers," Proceedings of the Conference on Industrial Electronics and Applications, IEEE Publ., Piscataway, NJ, 2009, pp. 913-918. doi:10.1109/ICIEA.2009.5138329

- [9] Silani, E., and Lovera, M., "Magnetic Spacecraft Attitude Control: A Survey and Some New Results," Control Engineering Practice, Vol. 13, No. 3, 2005, pp. 357-371. doi:10.1016/j.conengprac.2003.12.017

[10] Guiggiani, A., Kolmanovsky, I., Patrinos, P., and Bemporad, A., "FixedPoint Constrained Model Predictive Control of Spacecraft Attitude," Proceedings of the American Control Conference, IEEE Publ., 
Piscataway, NJ, 2015, pp. 2317-2322.

doi:10.1109/ACC.2015.7171078

-111] Crouch, P., "Spacecraft Attitude Control and Stabilization: Applications of Geometric Control Theory to Rigid Body Models," Transactions on Automatic Control, Vol. 29, No. 4, 1984, pp. 321-332. doi:10.1109/TAC.1984.1103519

-[12] Krishnan, H., McClamroch, H., and Reyhanoglu, M., "Attitude Stabilization of a Rigid Spacecraft Using Two Momentum Wheel Actuators," Journal of Guidance, Control, and Dynamics, Vol. 18, No. 2, 1995, pp. 256-263. doi: $10.2514 / 3.21378$

[13] Brockett, R. W., "Asymptotic Stability and Feedback Stabilization," Differential Geometric Control Theory, edited by Brockett, R. W., et al., Birkhauser Boston, Cambridge, MA, 1983, pp. 181-191.

[14] Byrnes, C. I., and Isidori, A., "On the Attitude Stabilization of Rigid Spacecraft," Automatica, Vol. 27, No. 1, 1991, pp. 87-95. doi:10.1016/0005-1098(91)90008-P

[15] Zabczyk, J., "Some Comments on Stabilizability," Applied Mathematics and Optimization, Springer-Verlag, New York, 1989, pp. 1-9.

[16] Gurvits, L., and Li, Z., "Smooth Time-Periodic Feedback Solutions for Nonholonomic Motion Planning," Progress in Nonholonomic Motion Planning, Springer-Verlag, New York, 1993, pp. 53-108.

[17] Horri, N. M., and Hodgart, S., "Attitude Stabilization of an Underactuated Satellite Using Two Wheels," Proceedings of the IEEE Aerospace Conference, Vol. 6, IEEE Publ., Piscataway, NJ, Nov. 2003, pp. 2629-2635. doi:10.1109/AERO.2003.1235188

[18] Horri, N. M., and Palmer, P., "Practical Implementation of AttitudeControl Algorithms for an Underactuated Satellite," Journal of Guidance, Control, and Dynamics, Vol. 35, No. 1, 2012, pp. 40-50. doi: $10.2514 / 1.54075$

[19] Leonard, N. E., and Krishnaprasad, P. S., "Averaging for Attitude Control and Motion Planning," Proceedings of the 32nd Conference on Decision and Control, Vol. 4, IEEE Publ., Piscataway, NJ, Dec. 1993, pp. 3098-3104. doi:10.1109/CDC.1993.325773

[20] Leonard, N. E., and Krishnaprasad, P. S., "Motion Control of Drift-Free, Left-Invariant Systems on Lie Groups," Transactions on Automatic Control, Vol. 40, No. 9, Sept. 1995, pp. 1539-1553. doi:10.1109/9.412625

[21] Yamada, K., Yoshiwa, S., and Yamaguchi, I., "Feedback Attitude Control of a Spacecraft by Two Reaction Wheels," Proceedings of the International Symposium on Space Technology and Science, Vol. 21, Agne Shofu Publishing Inc., Tokyo, 1998, pp. 550-555.

[22] Ge, X., and Chen, L., "Optimal Reorientation of Underactuated Spacecraft Using Genetic Algorithm with Wavelet Approximation," Acta Mechanica Sinica, Vol. 25, No. 4, 2009, pp. 547-553. doi:10.1007/s10409-009-0246-6

[23] Grüne, L., and Pannek, J., "Feasibility and Robustness," Nonlinear Model Predictive Control: Theory and Algorithms, edited by Isidori, A., et al., Springer-Verlag, New York, 2010, pp. 211-250.

[24] Meadows, E. S., Henson, M. A., Eaton, J. W., and Rawlings, J. B., "Receding Horizon Control and Discontinuous State Feedback Stabilization," International Journal of Control, Vol. 62, No. 5, 1995, pp. 1217-1229. doi:10.1080/00207179508921593

[25] De Oliveira Kothare, S. L., and Morari, M., "Contractive Model Predictive Control for Constrained Nonlinear Systems," Transactions on Automatic Control, Vol. 45, No. 6, 2000, pp. 1053-1071. doi:10.1109/9.863592

[26] Bemporad, A., Morari, M., Dua, V., and Pistikopoulos, E., "The Explicit Linear Quadratic Regulator for Constrained Systems," Automatica, Vol. 28, No. 1, 2002, pp. 3-20. doi:10.1016/S0005-1098(01)00174-1

[27] Hughes, P., "Rotational Kinematics," Spacecraft Attitude Dynamics, edited by Hughes, P., Dover, New York, 2004, pp. 18-28.

[28] Bloch, A., Reyhanoglu, M., and McClamroch, N. H., "Control and Stabilization of Nonholonomic Dynamic Systems," Transactions on Automatic Control, Vol. 37, No. 11, 1992, pp. 1746-1757. doi:10.1109/9.173144

[29] Bullo, F., "An Overview of Controllability for Control-Affine Systems," Geometric Control of Mechanical Systems, edited by Marsden, J. E., et al., Springer, New York, 2005, pp. 368-386.

[30] Sussmann, H. J., "A General Theorem on Local Controllability," Journal on Control and Optimization, Vol. 25, No. 1, 1987, pp. 158-194. doi:10.1137/0325011

[31] Jakubczyk, B., and Sontag, E. D., "Controllability of Nonlinear Discrete-Time Systems: A Lie-Algebraic Approach," Journal on Control and Optimization, Vol. 28, No. 1, 1990, pp. 1-33. doi:10.1137/0328001
[32] Albertini, F., and Sontag, E. D., "Further Results on Controllability Properties of Discrete-Time Nonlinear Systems," Dynamics and Control, Vol. 4, No. 3, edited by Ryan, E. P., Kluwer Academic, Norwell, MA, 1994, pp. 235-253. doi:10.1007/BF01985073

[33] Wei, L., and Byrnes, C. I., "Design of Discrete-Time Nonlinear Control Systems via Smooth Feedback," Transactions on Automatic Control, Vol. 39, No. 11, 1994, pp. 2340-2346. doi: $10.1109 / 9.333790$

[34] Goodwin, G. C., Seron, M. M., and De Doná, J. A., "Introduction to Constrained Control," Constrained Control and Estimation: An Optimisation Approach, edited by Sontag, E. D., et al., Springer-Verlag, New York, 2005, pp. 1-21.

[35] Petersen, C., Leve, F., and Kolmanovsky, I., "Hybrid Switching Attitude Control of Underactuated Spacecraft Subject to Solar Radiation Pressure," Proceedings of Spaceflight Mechanics Meeting, AAS/AIAA Paper 2015-327, 2015.

[36] Petersen, C., Leve, F., and Kolmanovsky, I., "Underactuated Spacecraft Switching Law for Two Reaction Wheels and Constant Angular Momentum," Journal of Guidance, Control, and Dynamics (to be published).

[37] Gilbert, E., and Kolmanovsky, I. V., "Nonlinear Tracking Control in Presence of State and Control Constraints: A Generalized Reference Governor," Automatica, Vol. 38, No. 12, 2002, pp. 2063-2073. doi:10.1016/S0005-1098(02)00135-8

[38] Walker, K., Samadi, B., Huang, M., Gerhard, J., Butts, K., and Kolmanovsky, I., "Design Environment for Nonlinear Model Predictive Control," SAE 2016 World Congress and Exhibition, Soc. of Automotive Engineers Paper 2016-01-0627, Warrendale, PA, 2016. doi: $10.4271 / 2016-01-0627$

[39] Pingree, P. J., "Advancing NASA's On-Board Processing Capabilities with Reconfigurable FPGA Technologies," Aerospace Technologies Advancements, edited by Arif, T. T., Intech, Rijeka, Croatia, 2010, pp. 69-86. doi: $10.5772 / 6938$

[40] Leve, F. A., Hamilton, B. J., and Peck, M. A., "Momentum Device Technology Tradeoffs," Spacecraft Momentum Control Systems, Vol. 1010, Springer-Verlag, New York, 2015, pp. 49-55. doi:10.1007/978-3-319-22563-0

[41] Leve, F. A., Hamilton, B. J., and Peck, M. A., "Momentum Control Devices and Attitude Control Systems," Spacecraft Momentum Control Systems, Vol. 1010, Springer-Verlag, New York, 2015, pp. 5-10. doi:10.1007/978-3-319-22563-0

[42] Diehl, M., Uslu, I., Findeisen, R., Schwarzkopf, S., Allgöwer, F., Bock, H. G., Bürner, T., Gilles, E. D., Kienle, A., Schlöder, J. P., and Stein, E., "Real-Time Optimization for Large Scale Processes: Nonlinear Model Predictive Control of a High Purity Distillation Column," Online Optimization of Large Scale Systems, Springer-Verlag, New York, 2001, pp. 363-383.

doi:10.1007/978-3-662-04331-8_20

[43] Diehl, M., Bock, H. G., Schlöder, J. P., Findeisen, R., Nagu, Z., and Allgöwer, F., "Real-Time Optimization and Nonlinear Model Predictive Control of Processes Governed by Differential-Algebraic Equations," Journal of Process Control, Vol. 12, No. 4, 2002, pp. 577-585. doi:10.1016/S0959-1524(01)00023-3

[44] Seron, M. M., Donà, J. A., and Goodwin, G. C., "Global Analytical Model Predictive Control with Input Constraints," Proceedings of the 39th Conference on Decision and Control, IEEE Publ., Piscataway, NJ, 2000, pp. 154-159. doi: $10.1109 /$ CDC. 2000.912749

[45] Tøndel, P., Johansen, T. A., and Bemporad, A., "An Algorithm for Multi-Parametric Quadratic Programming and Explicit MPC Solutions," Automatica, Vol. 39, No. 3, 2003, pp. 489-497. doi:10.1016/S0005-1098(02)00250-9

[46] Grieder, P., Borrelli, F., Torrisi, F., and Morari, M., "Computation of the Constrained Infinite Time Linear Quadratic Regulator,' Automatica, Vol. 40, No. 4, 2004, pp. 701-708. doi:10.1016/j.automatica.2003.11.014

[47] Dinh, Q. T., Savorgnan, C., and Diehl, M., "Adjoint-Based PredictorCorrector Sequential Convex Programming for Parametric Nonlinear Optimization," Journal on Optimization, Vol. 22, No. 4, 2012, pp. 1258-1284.

[48] Zavala, V. M., Laird, C. D., and Biegler, L. T., "A Fast Moving Horizon Estimation Algorithm Based on Nonlinear Programming Sensitivity," Journal of Process Control, Vol. 18, No. 9, 2008, pp. 876-884. doi:10.1016/j.jprocont.2008.06.003

[49] Kungurtsev, V., and Diehl, M., "SQP Methods for Parametric Nonlinear Optimization," Optimization Online, 2014, pp. 1-35. 
This article has been cited by:

1. Alexander Frias, Anton H. J. de Ruiter, Krishna Dev Kumar. Velocity-Free Attitude Stabilization of a Nadir-Pointing Underactuated Rigid Spacecraft. Journal of Guidance, Control, and Dynamics, ahead of print1-15. [Abstract] [Full Text] [PDF] [PDF Plus]

2. Bingheng Wang, Zhongjie Meng, Panfeng Huang. Nonlinear MPC Based Coordinated Control of Towed Debris Using Tethered Space Robot 698-706. [Crossref] 